\title{
THE EFFECTIVE ELECTRICAL CONDUCTIVITY OF NONLINEAR LAMINATE COMPOSITES
}

\author{
BY \\ GUOAN LI AND ANDREW S. DOUGLAS \\ Department of Mechanical Engineering, The Johns Hopkins University, Baltimore, Maryland
}

\begin{abstract}
This paper studies the effective nonlinear electrical conductivity of sequentially laminated materials in both two and three dimensions. The exact nonlinear conductive behavior is obtained through a variational procedure [1] that expresses the nonlinear properties of the laminate composite in terms of an optimization with respect to the properties of a series of linear comparison materials. Multiphase laminate composites are discussed and these results are compared with nonlinear HashinShtrikman (H-S) bounds. It is found that some of the laminates possess extremal microstructures that attain the nonlinear H-S lower bound while others are very close to extremal microstructures. The results apply also to the nonlinear thermal conductivity and dielectric and magnetic behavior of laminated heterogeneous materials.
\end{abstract}

I. Introduction. Even though the effective physical properties of composite materials have been studied for a long time, their complex microgeometry has made exact evaluation of these effective properties difficult. While a limited number of microgeometries, such as dilute and some periodic structures, have been solved for exactly, bounds on the properties and approximations to the composite behavior have provided useful information in the absence of a precise characterization of heterogeneous materials.

This paper focuses on the nonlinear electrical conductivity of laminated heterogeneous materials. The results obtained apply also to the thermal conductivity and the dielectric and magnetic behavior of heterogeneous media. In each of these physical problems, when the constituent phases are linear, the vector field gradients and fluxes are related by a second-rank tensor. The effective elastic response of heterogeneous materials is characterized by a fourth-rank tensor.

The study of the conductive behavior of composite materials dates back to Maxwell [2]. Notable contributions include work by Hashin and Shtrikman [3], Kerner [4], Willis [5], and others. Landauer [6] has an extensive list of references in this area. Broadly speaking, the different methods proposed to predict the effective properties of linear composite materials can be classified into three categories. The first category is based on identifying specific microstructures and obtaining the effective properties

Received November 11, 1992.

(C)1995 Brown University 
exactly. The solution to periodic microstructures belongs to this category. The second approach is to determine the range of possible behaviors for a given material class according to some known microstructural parameters. An important example of this approach is the Hashin-Shtrikman [3] (H-S) bounds on the electrical conductivity of composite materials. The third category postulates approximate models that capture the essential features of the microstructure of a given heterogeneous system. Effective medium theories [7] are examples of a commonly used method in this category.

It was first recognized by Bruggeman $[8,9]$ that the linear effective conductivity can be calculated exactly for a wide class of composites constructed through a laminating procedure. The "rank" of a laminate denotes the number of times a laminating process is used to form the composite. A rank-I laminate (see Fig. 1(a)) is constructed just by layering two materials in alternate order to form a multilayered sandwich. Higher-rank laminates have periodic particulate microstructures.

When the thickness of each successive laminate layer used to create the next rank laminate structure is sufficiently small, the physical properties of the resulting laminate composite material are easy to evaluate since the local fields are piecewise constant [9, 10]. Even though laminates of high rank are difficult to manufacture, as pointed out by Milton [10], they are important theoretical tools, which give insight into the properties of composites in general. Milton [10, 11] and Lurie and Cherkaev $[12,13]$ found that laminate materials are important not only because the exact evaluation could be obtained for their physical properties, but also because they attain many bounds on the complex physical properties (e.g., conductive constants, elastic moduli) of a general two-phase composite. Also, the rank-I nonlinear laminate (with both phase 1 and phase 2 made of nonlinear materials) has been solved exactly and this solution attains the nonlinear Reuss and Voigt bounds (see Ponte

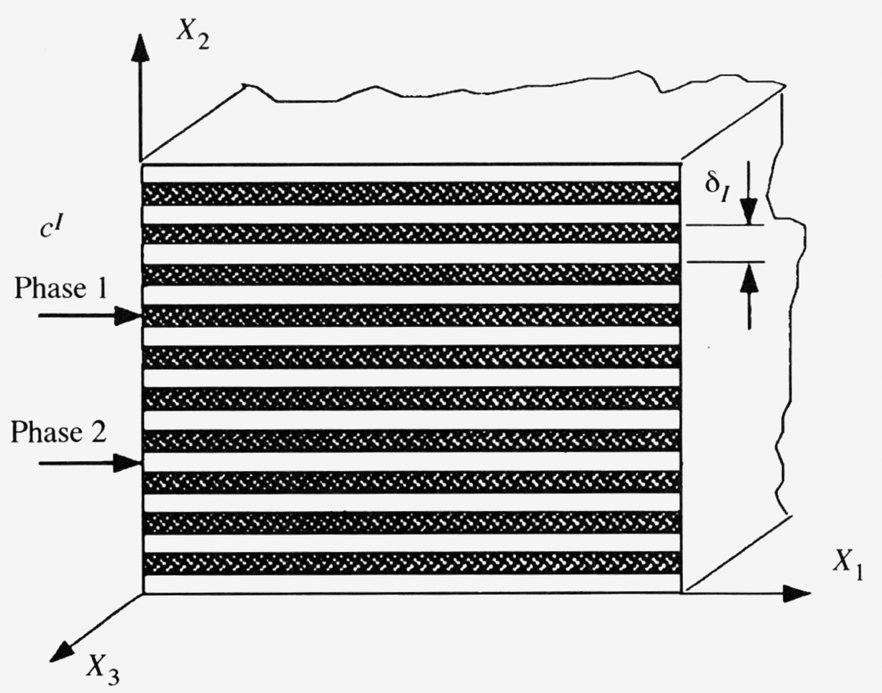

FIG. 1(a). Rank-I laminated material (laminated in the $x_{2}$ direction) 
Castañeda [14]). Thus, it is hoped that nonlinear laminate composites can be used as an effective way in which to obtain good estimates on the bounds and effective properties of composites.

In general, nonlinear heterogeneous materials present extreme difficulties in their analysis. Consequently, there are fewer approaches available for the study of nonlinear problems than for linear problems. There are, however, many electrical-transport phenomena in solids in which nonlinearity plays an important role. Such nonlinearities show up in effects such as dielectric breakdown and the burning out of fuses [15]. The design of nonlinear optical materials also presents an interesting application of nonlinear composite theory.

In the study of nonlinear composite materials, Willis [16] proposed an extension of the Hashin-Shtrikman variational principles to nonlinear composites. Talbot and Willis [17] used this method to calculate nonlinear bounds for effective properties of nonlinear heterogeneous dielectrics. Stroud and Hui [18] and Zeng et al. [15] studied the weakly nonlinear composite by means of the Taylor expansion. Ponte Castañeda [1] has proposed a new variational method in the study of the effective properties of nonlinear composites from which a strict lower bound and an estimate to the upper bound can be generated. This variational method is used here to calculate the exact effective electrical conductivity of nonlinear laminated materials that are isotropic in two-dimensional space or three-dimensional space.

The variational principle due to Ponte Castañeda $[1,14]$ is designed to yield the effective properties of nonlinear heterogeneous systems in terms of the effective properties of suitably optimized linear heterogeneous systems of the same microstructure. Thus, in order to make use of this variational procedure to evaluate the effective conductivity of nonlinear laminated materials, the exact evaluation of the effective properties of linear laminated materials is fully derived in Appendix A. The resulting nonlinear effective properties can then be compared with "nonlinear Hashin-Shtrikman bounds". These are H-S bounds because they are obtained by introducing the linear H-S bounds into Ponte Castañeda's variational principle [1, 14]. The generation of these nonlinear $\mathrm{H}-\mathrm{S}$ bounds is outlined in Appendix B.

In the following sections, the general theory describing electrical conductivity in multiphase composites is introduced and Ponte Castañeda's nonlinear variational procedure [1] is reviewed. Application of this variational procedure provides the exact solutions to sequentially laminated nonlinear composites.

Since most of the known results quantifying the nonlinear response of composite materials are approximations, exact solutions to a new class (laminated media) of nonlinear materials is important. On the basis of this work, it is evident that laminated materials can play the same important role in nonlinear composite theory as they do in linear composite theory.

\section{Effective properties and variational principles.}

A. Definition of effective properties. Consider an $N$-phase composite that has a volume $V$ and outer boundary $\partial V$. The electrical conductivity of the composite material is described by an energy density function of the form 


$$
w(\mathbf{x}, \mathbf{E})=\sum_{r=1}^{N} \chi^{(r)}(\mathbf{x}) W^{(r)}(\mathbf{E}),
$$

where $W^{(r)}$ is a function that grows faster than the quadratic magnitude of the vector $\mathbf{E}(E=|\mathbf{E}|)$, where the vector $\mathbf{E}$ represents the electric field, $\mathbf{x}$ is the Cartesian spatial coordinate, and $\chi^{(r)}(\mathbf{x})$ is the characteristic function for phase $r$ that occupies discontinuous volume $V^{(r)}$ and is expressed by

$$
\chi^{(r)}(\mathbf{x})= \begin{cases}1, & \text { if } \mathbf{x} \in V^{(r)}, \\ 0, & \text { otherwise. }\end{cases}
$$

If $c^{(r)}$ denotes the volume concentration of phase $r$, then $c^{(r)}$ is expressed as

$$
c^{(r)}=\frac{1}{V} \int_{V} \chi^{(r)}(\mathbf{x}) d V .
$$

Obviously, in an $N$-phase body, $\sum_{r=1}^{N} c^{(r)}=1$.

Let $\phi$ denote the electric field potential; then $\mathbf{E}$ is defined by

$$
\mathbf{E}=-\nabla \phi .
$$

The electric current density $\mathbf{j}$, which is a vector, is defined to be the derivative of the energy density function $w$ with respect to $\mathbf{E}$,

$$
\mathbf{j}=\frac{\partial w}{\partial \mathbf{E}} \text {. }
$$

For linear materials, $\mathbf{j}=\boldsymbol{\sigma} \cdot \mathbf{E}$, where $\boldsymbol{\sigma}$ is the second-order conductivity tensor, which, for isotropic materials has a single eigenvalue. Thus $\boldsymbol{\sigma}=\sigma \mathbf{I}$ (I is the identity tensor) and $\mathbf{j}=\sigma \mathbf{E}$, where $\sigma$ is the isotropic electrical conductivity constant.

The governing equation is derived from the fact that in a steady current, the spatial distribution of the curren $\mathbf{i}$ density, $\mathbf{j}$, is independent of time, and satisfies the equation

$$
\nabla \cdot \mathbf{j}=0 \text {. }
$$

At the interface between any two phases, the potential $\phi$ and the component of $\mathbf{j}$ normal to this interface must be continuous.

A uniform boundary condition on the outer boundary $\partial V$ is defined, as in Hill [19], by

$$
\phi(\mathbf{x})=-\overline{\mathbf{E}} \cdot \mathbf{x}, \quad \mathbf{x} \in \partial V,
$$

where $\overline{\mathbf{E}}$ is an arbitrary constant vector. It can easily be shown that the average electric field over the whole body is exactly $\overline{\mathbf{E}}$, i.e.,

$$
\overline{\mathbf{E}}=\frac{1}{V} \int_{V} \mathbf{E}(\mathbf{x}) d V .
$$

The average electrical current density, $\overline{\mathbf{j}}$, is defined in a similar way by

$$
\overline{\mathbf{j}}=\frac{1}{V} \int_{V} \mathbf{j}(\mathbf{x}) d V
$$


The purpose of this paper is to determine the effective nonlinear conductive properties of nonlinear laminate composites. This constitutive property is characterized by the effective energy density function $\widetilde{W}$ of the heterogeneous system, see Hill [19], which relates $\overline{\mathbf{E}}$ and $\overline{\mathbf{j}}$ through

$$
\overline{\mathbf{j}}=\frac{\partial \widetilde{W}}{\partial \overline{\mathbf{E}}}
$$

The effective nonlinear energy density function of the heterogeneous material, $\widetilde{W}$, can be obtained directly by using the minimum principle of energy density by means of

$$
\widetilde{W}(\overline{\mathbf{E}})=\min _{\mathbf{E} \in \mathbf{A}} \frac{1}{V} \int_{V} w(\mathbf{x}, \mathbf{E}(\mathbf{x})) d V,
$$

where $\mathbf{A}$ is the set of all admissible electric fields defined by

$$
\mathbf{A}=\{\mathbf{E} \mid \mathbf{E}=-\nabla \phi(\mathbf{x}) \text { in } V \text {, and } \phi=-\overline{\mathbf{E}} \cdot \mathbf{x} \text { on } \partial V\} .
$$

The energy density function, defined by Eq. (1), $w(\mathbf{x}, \mathbf{E}(\mathbf{x}))$, at each point of the heterogeneous material is obtained by solving the complete electrical conductivity problem as defined by Eqs. (4) through (7). However, for most real nonlinear heterogeneous systems, it is impractical to solve the electric field problem exactly due to its microstructural complexity and the nonlinear behavior of the system. The variational principle (11) is thus difficult to use.

In the following section we briefly review a variational procedure due to Ponte Castañeda [1], which addresses the effective nonlinear constitutive problem, and use it to evaluate the effective properties of nonlinear heterogeneous materials with specific microstructures. Detailed descriptions for linear electrical conductivity problems can be found in Landau [20].

B. Ponte Castañeda's variational procedure. We restrict our attention to heterogeneous materials in which each phase is isotropic. The energy function of the heterogeneous material can therefore be written as $w(\mathbf{x}, \mathbf{E})=f(\mathbf{x}, u)$ where $u=E^{2}$ ( $f$ has the same dependence on $\mathbf{x}$ as has $w$ ). We assume that $w$ grows faster than $E^{2}$-the function $f$ is therefore convex in the variable $u$.

Using Ponte Castañeda's variational principle $[1,14]$, the energy density function at each point of the heterogeneous material is written

$$
w(\mathbf{x}, \mathbf{E})=\max _{\sigma_{0}(\mathbf{x})>0}\left\{w_{0}(\mathbf{x}, \mathbf{E})-v\left(\mathbf{x}, \sigma_{0}\right)\right\},
$$

where

$$
w_{0}(\mathbf{x}, \mathbf{E})=\frac{1}{2} \sigma_{0}(\mathbf{x}) E^{2} \quad \text { and } \quad v\left(\mathbf{x}, \sigma_{0}(\mathbf{x})\right)=\max _{E}\left\{w_{0}(\mathbf{x}, \mathbf{E})-w(\mathbf{x}, \mathbf{E})\right\},
$$

where $w_{0}(\mathbf{x}, \mathbf{E})$ corresponds to the local energy density function of a linear, heterogeneous comparison material with an arbitrary nonnegative electrical conductivity $\sigma_{0}(\mathbf{x})$. Basically, $v\left(\mathbf{x}, \sigma_{0}(\mathbf{x})\right)$ is just a Legendre dual of the function $f$, and $w(\mathbf{x}, \mathbf{E})$ in (13) is nothing more than a second Legendre transformation for $f$. For more details, the reader is referred to Ponte Castañeda [1]. 
The effective energy function of the composite, $\widetilde{W}(\overline{\mathbf{E}})$, is given by the volume average of $w(\mathbf{x}, \mathbf{E})$ in (13), namely,

$$
\widetilde{W}(\overline{\mathbf{E}})=\max _{\sigma_{0}(\mathbf{x})>0}\left\{\widetilde{W}_{0}(\overline{\mathbf{E}})-\frac{1}{V} \int_{V} v\left(\mathbf{x}, \sigma_{0}(\mathbf{x})\right) d V\right\} .
$$

Here $\widetilde{W}_{0}(\overline{\mathbf{E}})$ denotes the effective energy function of the linear heterogenerous comparison material with local electrical conductivity $\sigma_{0}(\mathbf{x})$, and is given by

$$
\widetilde{W}_{0}(\overline{\mathbf{E}})=\min _{\mathbf{E} \in \mathbf{A}} \frac{1}{V} \int_{V} w_{0}(\mathbf{x}, \mathbf{E}) d V .
$$

Equation (15) is an alternative way to determine the effective energy function of nonlinear composites in terms of the effective energy function of a suitably optimized linear comparison heterogeneous material. It should be emphasized that the electrical conductivity $\sigma_{0}(\mathbf{x})$ in Eq. (15) is an arbitrary nonnegative function. In this sense Eq. (15) leads to an infinite-dimensional optimization over a nonnegative function space. Thus, in practice, it might seem impossible to obtain the optimal linear comparison material, i.e., to define $\sigma_{0}(\mathbf{x})$ in $V$ such that (15) is satisfied. However, it follows from (14) that, if the electric field $\mathbf{E}$ is constant within any region, say $V^{(r)} \in$ $V$, then $\sigma_{0}(\mathbf{x})=\sigma_{0}^{(r)}$ in $V^{(r)}$, where $\sigma_{0}^{(r)}$ is the constant conductivity in $V^{(r)}$. This property of the electric field reduces (15) to an optimization with respect to a piecewise constant function $\sigma_{0}^{(r)}$. Under this condition, it may be possible to obtain an evaluation of the effective nonlinear conductivity characterized by $\widetilde{W}$. The dual version of (15) can also be derived in terms of the minimum principle of complementary energy as shown by Ponte Castañeda [1].

III. Application of the variational principle to multiphase laminated materials. In this section, we study a specific type of microstructure-sequentially laminated materials which, when subjected to the uniform boundary condition defined by Eq. (7), have a piecewise-constant electric field over the whole material. In this case, the variational principle (15) makes it possible to obtain an exact solution to the effective nonlinear energy function $\widetilde{W}$. By choosing the volume concentrations of a sequentially laminated linear material comprised of two isotropic constituents appropriately, the (linear) composite behaves isotropically in response to an applied electric field (Lurie and Cherkaev $[12,13])$. The effective conductivity of this laminated material attains the corresponding (linear) H-S bounds (Lurie and Cherkaev [12, 13]). In this work, we examine the effective conductivity of sequentially laminated materials that are composed of two nonlinear constituents. The solutions for linear laminated materials are used as comparison materials for the nonlinear laminated materials.

The construction of a laminated material always begins by slicing the two components and layering these slices with each other in an alternate order to form a rank I laminated material, as shown in Fig. 1(a). The scale of the thickness of each layer is small compared with the material specimen dimension $l$. Thus, for the rank-I laminate in Fig. 1(a), the thickness of each layer $\delta_{I} \ll l$. The direction normal to the layer plane is defined as the laminate direction. For example, in Fig. 1(a), $x_{2}$ is the direction of the rank-I laminate. If this laminate is then sliced on a length scale $\delta_{I I}$ 
in a different direction from the previous slices, and layered with slices from another material in an alternate order, a rank-II laminated material is formed, as shown in Figs. $1(\mathrm{~b})$ and $1(\mathrm{c})$, where $\delta_{I} \ll \delta_{I I} \ll l$. This laminating procedure can be repeated any number of times and in any direction to construct a hierarchical microstructure.

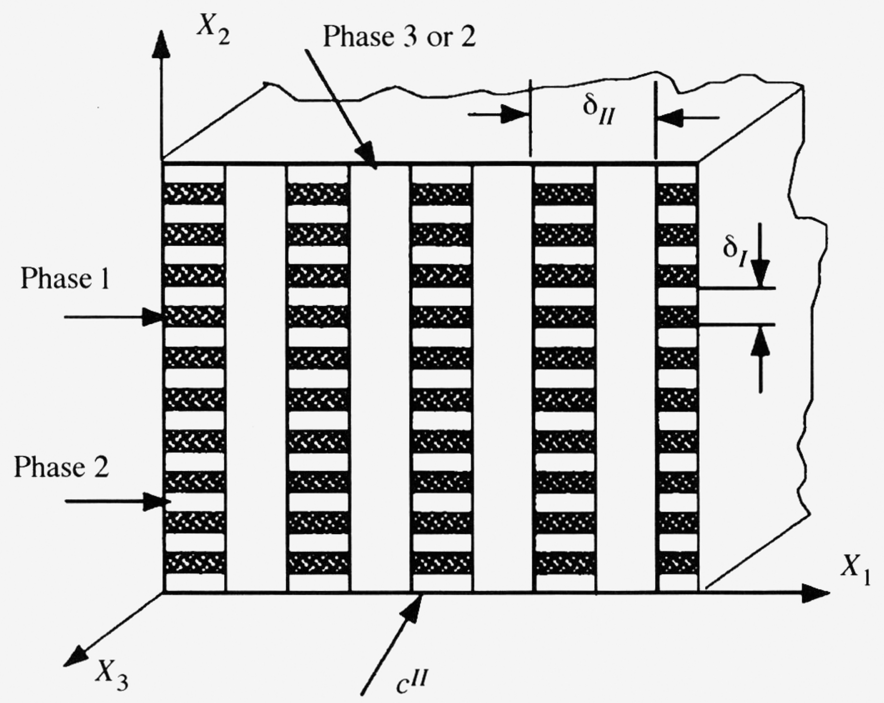

FIG. 1(b). Rank-II laminated material (laminated in the $x_{1}$ direction, $\left.\delta_{I I} \gg \delta_{I}\right)$

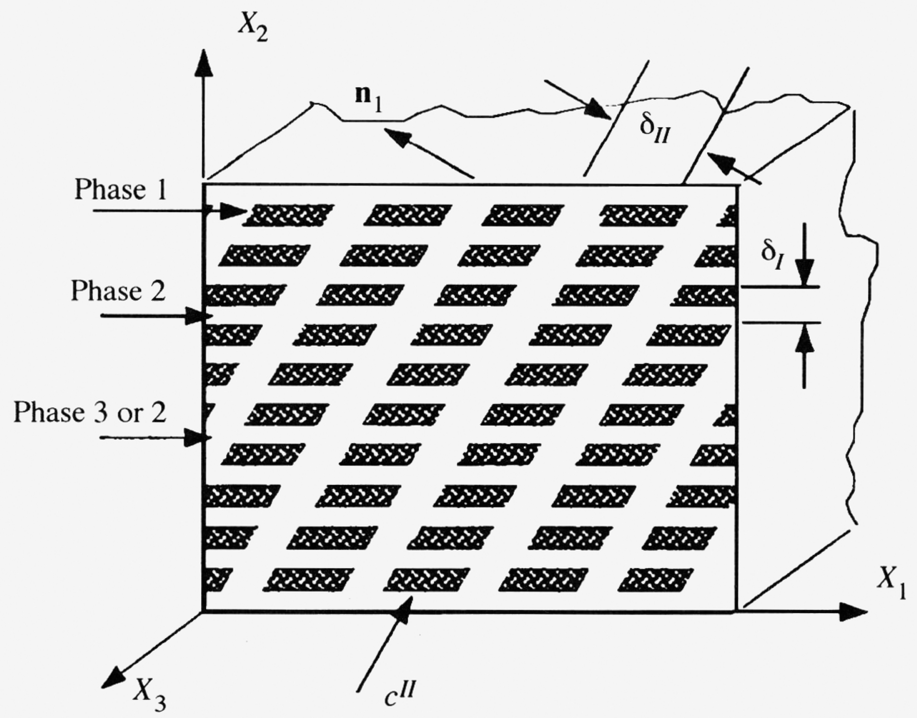

FIG. 1(c). Rank-II laminated material (laminated in the $\mathbf{n}_{1}$ direction, $\left.\delta_{I I} \gg \delta_{I}\right)$ 
A key aspect in using the variational procedure (15) to calculate the effective energy function of nonlinear, sequentially laminated materials is the existence of the effective energy function $\widetilde{W}_{0}$ of a linear, sequentially laminated material of identical microstructure. We therefore briefly review the derivation of the effective conductivity of linear composites with this kind of microstructure.

Consider a laminated material comprised of two linear components. If one component has an anisotropic conductivity tensor, $\sigma^{(1)}$, and the other component is isotropic with conductivity $\sigma_{0}^{(2)} \mathbf{I}$, then the laminate composite made by laminating these two components in direction $\mathbf{n}$ has an anisotropic electrical conductivity, given by $[21]$,

$$
\overline{\boldsymbol{\sigma}}_{0}=\sigma_{0}^{(2)} \mathbf{I}+c^{(1)}\left(\boldsymbol{\sigma}^{(1)}-\sigma_{0}^{(2)} \mathbf{I}\right)-\frac{c^{(1)} c^{(2)}\left(\boldsymbol{\sigma}^{(1)}-\sigma_{0}^{(2)} \mathbf{I}\right) \mathbf{n} \otimes\left(\boldsymbol{\sigma}^{(1)}-\sigma_{0}^{(2)} \mathbf{I}\right) \mathbf{n}}{\sigma_{0}^{(2)}+c^{(2)}\left[\left(\boldsymbol{\sigma}^{(1)}-\sigma_{0}^{(2)} \mathbf{I}\right) \mathbf{n}\right] \mathbf{n}}
$$

where $c^{(1)}, c^{(2)}\left(c^{(1)}+c^{(2)}=1\right)$ are the volume fractions of component 1 and component 2 , respectively. This equation can be used repetitively to obtain the effective properties of higher-rank laminates, as long as one phase is isotropic at each step in the laminating process. In this paper, a rank- $N$ laminate is composed of slices cut from a rank- $(N-1)$ laminate and an isotropic material. The volume fraction (which will be called the relative volume fraction later) of the rank- $(N-1)$ laminate is denoted $c^{N}$ in this rank- $N$ laminated material. For example, $c^{I}$ denotes the relative volume fraction of phase 1 in the rank-I laminate, and $c^{I I}$ denotes the relative volume fraction of the rank-I laminate within a rank-II laminate. It is seen that, in general, the resulting effective electrical conductivity $\bar{\sigma}_{0}$ from (17) is not isotropic. However, this anisotropic material can be made isotropic by requiring that the diagonal terms of $\bar{\sigma}_{0}$ be identical and that the off-diagonal terms vanish, i.e.,

$$
\begin{array}{ll}
\left(\overline{\boldsymbol{\sigma}}_{0}\right)_{i j}=0, & \text { if } i \neq j ; \\
\left(\overline{\boldsymbol{\sigma}}_{0}\right)_{i j}=\bar{\sigma}_{0}, & \text { if } i=j,
\end{array}
$$

where $\overline{\boldsymbol{\sigma}}_{0}$ is the effective isotropic conductivity of the linear laminated material. Equation (18) effectively selects the specific volume fractions for each phase $\left(c^{N}, \ldots\right.$, $c^{I}$ ) of the laminated material, which ensures an effective isotropic response.

The effective energy function of the linear, isotropic comparison material $\widetilde{W}_{0}$ is then given by

$$
\widetilde{W}_{0}(\overline{\mathbf{E}})=\frac{1}{2}\left(\overline{\boldsymbol{\sigma}}_{0} \overline{\mathbf{E}}\right) \cdot \overline{\mathbf{E}}=\frac{1}{2} \bar{\sigma}_{0} \bar{E}^{2} .
$$

Based on the fact that the electric field (in these specially constructed laminate composites) is piecewise constant in each phase, an exact solution for the effective energy function $\widetilde{W}$ of a nonlinear, isotropic laminated material can be obtained by using the variational procedure (15) with $\widetilde{W}_{0}$ defined by (19) as the linear comparison energy function. Note that, in this case, the maximizing comparison conductivity function $\sigma_{0}(\mathbf{x})$ in $(15)$ is constant within each phase. The variational procedure therefore leads to

$$
\widetilde{W}(\overline{\mathbf{E}})=\max _{\sigma_{0}^{(1)} \ldots \sigma_{0}^{(N)}}\left\{\widetilde{W}_{0}(\overline{\mathbf{E}})-\sum_{r=1}^{N} c^{(r)} v^{(r)}\left(\sigma_{0}^{(r)}\right)\right\},
$$


where $c^{(r)}$ is the volume fraction of phase $r$, while $v^{(r)}$ is defined by

$$
v^{(r)}\left(\sigma_{0}^{(r)}\right)=\max _{E^{2}}\left\{\frac{1}{2} \sigma_{0}^{(r)} E^{2}-W^{(r)}(\mathbf{E})\right\} .
$$

The maximum of the difference in $(21)$ is well bounded since $W^{(r)}$ grows faster than $E^{2}$.

The effective energy function obtained from (20) characterizes the overall electrical conductivity of an isotropic laminated material when the individual constituent materials (or phases) are nonlinear. It is noted that a sequentially laminated material represents a periodic particulate microstructure.

The rank-II laminate shown in Fig. 1(b) has fibers of rectangular cross section (which are long in the $x_{3}$ direction) in a periodic array in the $\left(x_{1}, x_{2}\right)$-plane. Figure 1 (c) shows another microstructure that is periodic in the $\left(x_{1}, x_{2}\right)$-plane, but with parallelogram fibers. The resulting microstructures consist of a continuous phase (the matrix) in which there is a regular distribution of noncontiguous inclusions. For example, in Figs. 1(b) and 1(c), both phase 2 and phase 3 are made of the identical matrix material, whereas phase 1 is made of the inclusion material.

It should be pointed out that, even if two "phases" are comprised of the same material, if the electric field $\mathbf{E}$ is different in each region, then these two layers must be treated as two different phases when using the variational principle (20), since they may have different linear comparison conductivities $\sigma_{0}^{(r)}$. For example, if phase 2 and phase 3 of the laminate in Fig. 1(b) are made of the same nonlinear material, the corresponding linear comparison conductivities $\sigma_{0}^{(2)}$ and $\sigma_{0}^{(3)}$ may be different. Hence, the nonlinear material is still treated as a three-phase composite, even though it is made of two constituents.

IV. The effective properties of nonlinear laminates. From the above analysis we see that the first step in computing the exact energy function for a nonlinear laminated material is to evaluate the effective electrical conductivity of the linear comparison material. Several laminates have been constructed in Appendix A based on the procedure described in the previous section. They are used as comparison materials to study the effective nonlinear electric conductivities of two- and three-dimensional isotropic heterogeneous materials constructed through a sequential laminating process.

Consider a nonlinear isotropic rank- $N$ laminated material consisting of $N+1$ phases, each phase being characterized by a potential function

$$
W^{(r)}(\mathbf{E})=\frac{1}{2} \sigma^{(r)} E^{2}+\frac{1}{n_{r}+1} \omega^{(r)} E^{n_{r}+1}, \quad r=1, \ldots, N+1,
$$

where $\sigma^{(r)}, \omega^{(r)}$ are linear and nonlinear isotropic conductivity constants and $n_{r}$ is the nonlinear order $\left(1<n_{r}<\infty\right)$ of phase $r$. The material characterized by $(22)$ is the most general form of material behavior considered here. The examples presented below use either this form or special cases derived from it. If $\omega^{(r)}=0$, phase $r$ is linear; if $\sigma^{(r)}=0$, phase $r$ is called a pure power-law material. 
Thus, on substituting the general nonlinear potential function (22) into (21) we obtain

$$
v^{(r)}\left(\sigma_{0}^{(r)}\right)=\frac{n_{r}-1}{2\left(n_{r}+1\right)}\left(\sigma_{0}^{(r)}-\sigma^{(r)}\right)^{\left(n_{r}+1\right) /\left(n_{r}-1\right)}\left(\omega^{(r)}\right)^{-2 /\left(n_{r}-1\right)} H\left(\omega^{(r)}\right),
$$

where $H\left(\omega^{(r)}\right)$ is a unit step function defined by

$$
H\left(\omega^{(r)}\right)= \begin{cases}0 & \text { if } \omega^{(r)}=0 \\ 1 & \text { if } \omega^{(r)}>0\end{cases}
$$

and

$$
\sigma_{0}^{(r)}=\sigma^{(r)} \quad \text { if } \omega^{(r)}=0
$$

The variational principle (20) can thus be used with (23) to yield the effective energy function of the nonlinear isotropic laminate, $\widetilde{W}$, viz.,

$$
\widetilde{W}(\overline{\mathbf{E}})=\max _{\sigma_{0}^{(r)}}\left\{\widetilde{W}_{0}(\overline{\mathbf{E}})-\sum_{r=1}^{N+1} c^{(r)} \frac{n_{r}-1}{2\left(n_{r}+1\right)}\left(\sigma_{0}^{(r)}-\sigma^{(r)}\right)^{\left(n_{r}+1\right) /\left(n_{r}-1\right)}\left(\omega^{(r)}\right)^{-2 /\left(n_{r}-1\right)} H\left(\omega^{(r)}\right)\right\} .
$$

In the following subsections we study different microstructures using the above procedure: first for two-dimensional laminates and then for three-dimensional laminates.

A. Nonlinear two-dimensional laminates. In this subsection, we consider electrical conduction in the $\left(x_{1}, x_{2}\right)$-plane. We restrict our attention to a nonlinear twophase composite comprised of one linear phase and one pure power-law constituent. The potential functions characterizing these constituents are given respectively by

$$
W^{(1)}(\mathbf{E})=\frac{1}{2} \sigma^{(1)} E^{2}, \quad W^{(2)}(\mathbf{E})=\frac{1}{n+1} \omega^{(2)} E^{n+1},
$$

where $\sigma^{(1)}, \omega^{(2)}, n$ are material constants, and $n>1$. Compared with (22), we see that $\omega^{(1)}=0, \sigma^{(2)}=0$, and $n_{2}=n$.

Hashin-Shtrikman (H-S) bounds on the effective conductivity of linear heterogeneous materials are based only on the volume concentration and properties of the constituent phases, without any knowledge of the microstructural details of the material. These bounds may be used in conjunction with the variational principle (15) to provide "nonlinear H-S bounds" as outlined briefly in Appendix B (see, Ponte Castañeda [1] for details).

Consider the two-dimensional nonlinear H-S bounds for the effective nonlinear energy function $\widetilde{W}$ of two-phase composites comprised of two constituents $W^{(1)}$ (with concentration $c^{(1)}$ ) and $W^{(2)}$ (with concentration $c^{(2)}$ ) as obtained from (87) of Appendix B. Two nonlinear bounds are obtained, one corresponding to the dispersed phase having greater conductivity, the other to the dispersed phase having smaller conductivity than the continuous phase. The two nonlinear bounds obtained result in bounds on the energy function given by

$$
W_{\mathrm{HS}}(\overline{\mathbf{E}})=\frac{1}{2} \max _{\sigma_{0}^{(2)}>0}\left[\left(\bar{\sigma}_{0}\right)_{i} \bar{E}^{2}-\frac{n-1}{n+1} c^{(2)}\left(\sigma_{0}^{(2)}\right)^{(n+1) /(n-1)}\left(\omega^{(2)}\right)^{-2 /(n-1)}\right],
$$


where $\left(\vec{\sigma}_{0}\right)_{i}$ is defined either by

$$
\left(\bar{\sigma}_{0}\right)_{i}=\sigma_{0}^{(1)}+\frac{2 c^{(2)} \sigma_{0}^{(1)}\left(\sigma_{0}^{(2)}-\sigma_{0}^{(1)}\right)}{c^{(1)} \sigma_{0}^{(2)}+\left(2-c^{(2)}\right) \sigma_{0}^{(1)}} \text { such that } W_{\mathrm{HS} 1}=W_{\mathrm{HS}},
$$

or by

$$
\left(\bar{\sigma}_{0}\right)_{i}=\sigma_{0}^{(2)}+\frac{2 c^{(1)} \sigma_{0}^{(2)}\left(\sigma_{0}^{(1)}-\sigma_{0}^{(2)}\right)}{c^{(2)} \sigma_{0}^{(1)}+\left(2-c^{(1)}\right) \sigma_{0}^{(2)}} \text { such that } W_{\mathrm{HS} 2}=W_{\mathrm{HS}} .
$$

In each case $\sigma_{0}^{(1)}=\sigma^{(1)}$ (because the phase 1 material is linear), $c^{(1)}$ and $c^{(2)}$ are the volume fractions of $W^{(1)}$ and $W^{(2)}$, respectively, and $W_{\mathrm{HS} 1}$ and $W_{\mathrm{HS} 2}$ are the two bounds corresponding to whether or not the more conductive material was the dispersed or continuous phase. The smaller of these two bounds is the strict nonlinear $\mathrm{H}-\mathrm{S}$ lower bound $\left(W_{\mathrm{HS}_{-}}\right)$, while the larger of these two bounds is an estimate to the $\mathrm{H}-\mathrm{S}$ upper bound (we still write this as $W_{\mathrm{HS}+}$ but should be mindful that this "upper estimate" is lower than the true upper bound). Thus,

$$
W_{\mathrm{HS}-}=\min \left(W_{\mathrm{HS} 1}, W_{\mathrm{HS}_{2}}\right), \quad W_{\mathrm{HS}+}=\max \left(W_{\mathrm{HS}_{1}}, W_{\mathrm{HS}_{2}}\right) .
$$

(a) Rank-II laminates. As the first example, consider a rank-II laminate that is isotropic in the $\left(x_{1}, x_{2}\right)$-plane. This laminate is composed of linear and pure powerlaw phases and has a microstructure in which long (in the $x_{3}$ direction) rectangular fibers are periodic in the $\left(x_{1}, x_{2}\right)$-plane (see Fig. 1(b)). The appropriate linear comparison material is the linear rank-II laminate discussed in Appendix A. This material (in three dimensions) is transversely isotropic with respect to the $x_{3}$ direction. In the $\left(x_{1}, x_{2}\right)$-plane, the effective isotropic conductivity $\bar{\sigma}_{0}^{I I}$ of the linear comparison material is given by (67) subject to the isotropy condition (68). The effective energy of this linear comparison laminate is thus given by

$$
\widetilde{W}_{0}(\overline{\mathbf{E}})=\frac{1}{2} \bar{\sigma}_{0}^{I I} \bar{E}^{2}
$$

Two different nonlinear composites can be constructed in this manner. One has a nonlinear matrix with linear inclusions, the other has a linear matrix with nonlinear inclusions. Consider the first of these two laminate composites. Thus the inclusions (phase 1 only in Fig. 1(b)) are described by $W^{(1)}$, and the matrix (which is composed of both phase 2 and phase 3 in Fig. 1(b)), is described by the nonlinear potential $W^{(2)}$. Using the variational principle (26), we can write the optimization equation that gives the exact effective properties of the isotropic laminate, $\widetilde{W}$, if the isotropy condition (68) is satisfied. However, it is useful to nondimensionalize the effective potential, $\widetilde{W}$, with respect to the linear material $W^{(1)}$. Thus

$$
\frac{\widetilde{W}(\overline{\mathbf{E}})}{W^{(1)}}=\max _{\substack{\sigma_{0}^{(2)}>0 \\ 1>c^{\prime}>c^{(1)}}}\left[\frac{\bar{\sigma}_{0}^{I I}}{\sigma^{(1)}}-F \frac{n-1}{n+1}\left(\frac{\omega^{(2)} \bar{E}^{n-1}}{\sigma^{(1)}}\right)^{-2 /(n-1)}\right],
$$

with $F$ defined by

$$
F=c^{I I}\left(1-c^{I}\right)\left(\frac{\sigma_{0}^{(2)}}{\sigma^{(1)}}\right)^{(n+1) /(n-1)}+\left(1-c^{I I}\right)\left(\frac{\sigma_{0}^{(3)}}{\sigma^{(1)}}\right)^{(n+1) /(n-1)} .
$$


The isotropy condition that constrains this optimization is given by

$$
\begin{aligned}
& c^{I I}\left(1-c^{I I}\right) A_{1}\left(\frac{\sigma_{0}^{(3)}}{\sigma^{(1)}}\right)^{2}+\left[\left(c^{I I}\right)^{2} \frac{\sigma_{0}^{(1)}}{\sigma^{(1)}}+c^{I I}\left(c^{I I}-2\right) A_{1} A_{2}\right] \frac{\sigma_{0}^{(3)}}{\sigma^{(1)}} \\
&+c^{I I}\left(1-c^{I I}\right) A_{1} \frac{\sigma_{0}^{(1)}}{\sigma^{(1)}}=0,
\end{aligned}
$$

where $c^{I I} c^{I}=c^{(1)}, \sigma_{0}^{(1)}=\sigma^{(1)}$ (because the inclusions are linear), and

$$
A_{1}=c^{I} \frac{\sigma_{0}^{(1)}}{\sigma^{(1)}}+\left(1-c^{I}\right) \frac{\sigma_{0}^{(2)}}{\sigma^{(1)}}, \quad A_{2}=c^{I} \frac{\sigma_{0}^{(2)}}{\sigma^{(1)}}+\left(1-c^{I}\right) \frac{\sigma_{0}^{(1)}}{\sigma^{(1)}} .
$$

This optimization was carried out using IMSL numerical routines. For this, rankII, two-dimensional composite optimization requires variation of the conductivities of the two linear comparison phases $\left(\sigma_{0}^{(2)}\right.$ for phase $2, \sigma_{0}^{(3)}$ for phase 3 ) which make up the nonlinear matrix material. However, the isotropy constraint (35) provides a relationship between $\sigma_{0}^{(2)}$ and $\sigma_{0}^{(3)}$, which is dependent on the microstructure through the relative concentrations $c^{I}, c^{I I}$. It was numerically expedient to optimize over $\sigma_{0}^{(2)}$ and the concentration $c^{I}$ (since $c^{I}$ is in a specific domain) and then use (35) to define $\sigma_{0}^{(3)}$ giving an isotropic response.

The second, rank-II, two-dimensional laminate has a linear matrix and nonlinear inclusions. Since, in this case, both phase 2 and phase 3 are made of the same linear material, $\sigma_{0}^{(2)}=\sigma_{0}^{(3)}=\sigma^{(1)}$. The isotropy constraint (35) determines the concentrations $c^{I}$ (thus $c^{I I}$ ), and the effective property is given by an optimization only with respect to $\sigma_{0}^{(1)}$, the conductivity of inclusions of the linear comparison material. This leads to an optimization expression identical to the nonlinear H-S bound (30), i.e.,

$$
\widetilde{W}(\overline{\mathbf{E}})=W_{\mathrm{HS} 2}(\overline{\mathbf{E}})
$$

(b) Rank-III two-dimensional laminates. Rank-III, two-dimensional laminates consist of layers cut from the rank-II laminate of Fig. 1(c) and layers from an isotropic material. (Sheets from the rank-II laminate material are cut along planes with normal in the $\left(x_{1}, x_{2}\right)$-plane.) The sheets of these two materials are laminated together in alternate order, as described in Appendix A. The resulting material has a microstructure with fibers (long in the $x_{3}$ direction) of trapezoidal and parallelogram cross sections arranged in a periodic manner in the $\left(x_{1}, x_{2}\right)$-plane. Appendix A gives both the conductivity of the linear, isotropic comparison material, $\bar{\sigma}_{0}^{I I I}$ in Eq. (78), and the isotropy conditions, in (77). The effective comparison energy function is thus given by

$$
\widetilde{W}_{0}(\overline{\mathbf{E}})=\frac{1}{2} \bar{\sigma}_{0}^{I I I} \bar{E}^{2} .
$$

There are two ways to construct this rank-III laminate microstructure with the two materials characterized by (27). The first uses linear inclusions (with potential $W^{(1)}$ and volume fraction $c^{(1)}$ ) in a nonlinear matrix (described by $W^{(2)}$ with volume concentration $c^{(2)}$ ). The application of the variational principle in Eqs. (23) and 
(26) gives $\sigma_{0}^{(1)}=\sigma^{(1)}$, and the effective energy function of the nonliner $x$ material

$$
\frac{\widetilde{W}(\overline{\mathbf{E}})}{W^{(1)}}=\max _{\substack{\sigma_{0}^{(2)}>0 \\ 1>c^{I}, c^{I I}>c^{(1)}}}\left[\frac{\bar{\sigma}_{0}^{I I I}}{\sigma^{(1)}}-F \frac{n-1}{n+1}\left(\frac{\omega^{(2)} \bar{E}^{n-1}}{\sigma^{(1)}}\right)^{-2 /(n-1)}\right],
$$

with $F$ defined by

$$
\begin{aligned}
F=c^{I I I} c^{I I}\left(1-c^{I}\right)\left(\frac{\sigma_{0}^{(2)}}{\sigma^{(1)}}\right)^{(n+1) /(n-1)}+c^{I I I}\left(1-c^{I I}\right)\left(\frac{\sigma_{0}^{(3)}}{\sigma^{(1)}}\right)^{(n+1) /(n-1)} \\
+\left(1-c^{I}\right)\left(\frac{\sigma_{0}^{(4)}}{\sigma^{(1)}}\right)^{(n+1) /(n-1)} .
\end{aligned}
$$

The isotropy conditions in (77) that constrain the above optimization are

$$
\begin{gathered}
\left(1-c^{I I}\right)\left(\sigma_{0}^{(3)}\right)^{2}-\left[2 \bar{\sigma}_{11}^{I}-c^{I I}\left(\bar{\sigma}_{11}^{I}+\bar{\sigma}_{22}^{I}\right)\right] \sigma_{0}^{(3)}+\left(1-c^{I I}\right) \bar{\sigma}_{11}^{I} \bar{\sigma}_{22}^{I}=0, \\
-\frac{1}{2}\left(\sigma_{0}^{(4)}\right)^{2}+\left[\frac{c^{I I I}}{1-c^{I I I}}\left(\bar{\sigma}_{11}^{I I}-\bar{\sigma}_{22}^{I I}\right)+\sqrt{3} B_{1}-B_{2}\right] \sigma_{0}^{(4)} \\
+\frac{S_{2}}{1-c^{I I I}}\left(\bar{\sigma}_{11}^{I I}-\bar{\sigma}_{22}^{I I}\right)+\left(B_{2}\right)^{2}-\left(B_{1}\right)^{2}=0,
\end{gathered}
$$

with

$$
\begin{gathered}
S_{2}=\frac{\left(1-c^{I I I}\right)}{4}\left(3 \bar{\sigma}_{11}^{I I}+\bar{\sigma}_{22}^{I I}+2 \sqrt{3} \bar{\sigma}_{12}^{I I}\right), \\
B_{1}=\frac{\sqrt{3}}{2} \bar{\sigma}_{11}^{I I}+\frac{1}{2} \bar{\sigma}_{12}^{I I}, \\
B_{2}=\frac{1}{2} \bar{\sigma}_{22}^{I I}+\frac{\sqrt{3}}{2} \bar{\sigma}_{12}^{I I},
\end{gathered}
$$

where $\bar{\sigma}_{11}^{I I}, \bar{\sigma}_{22}^{I I}$ are defined in Appendix A by Eq. (69) and $c^{I I I} c^{I I} c^{I}=c^{(1)}$. An iterative procedure is used for the above optimization. For a specified applied overall electric field $\overline{\mathbf{E}}$, Eqs. (41) and (42) are solved to obtain $\sigma_{0}^{(3)}$ and $\sigma_{0}^{(4)}$ for the initial estimates of $\sigma_{0}^{(2)}, c^{I}$, and $c^{I I}$. These are substituted into Eq. (39) to optimize for a new group of $\sigma_{0}^{(2)}, c^{I}$, and $c^{I I}$. Equations (41) and (42) are solved again to obtain a new group of $\sigma_{0}^{(3)}$ and $\sigma_{0}^{(4)}$. This iteration continues until convergence.

The second rank-III laminate is composed of a linear matrix described by $W^{(1)}$ and nonlinear inclusions described by $W^{(2)}$. Once again, the effective properties of this two-dimensional laminate coincide identically with the nonlinear $\mathrm{H}-\mathrm{S}$ bound $W_{\mathrm{HS} 2}$, defined in Eq. (30).

Note that, in two-dimensional space, the rank-II laminate depicted in Fig. 1(b) is the lowest "rank" that can manifest isotropy in the $\left(x_{1}, x_{2}\right)$-plane. While this laminate has a simple microstructure, higher-rank laminates have more complex microstructures. 
B. Nonlinear three-dimensional laminates. The effective properties of the twodimensional laminates obtained above illustrate the general manner in which exact properties can be obtained for nonlinear laminate composites. However, threedimensional properties (since $\overline{\mathbf{E}}$ may be arbitrarily oriented in space) are of greater practical significance. Although a very wide range of materials falls within the class of materials discussed here, we restrict our attention to two composite materials: (a) composites with linear and linear plus power-law constituents; (b) composites with both phases being pure power-law materials.

A rank-III, three-dimensional, laminate is constructed by layering slices cut from a rank-II laminate (see Fig. 1(b)) with slices from another isotropic material in alternate order. The slices from the rank-II laminate are cut parallel to the $\left(x_{1}, x_{2}\right)$-plane. These planes are layered with an isotropic phase in the $x_{3}$ direction to create the rank-III, three-dimensional, laminate. Let phase 1 be the inclusion phase and let the other three phases be made of the same material. The resulting composite has a microstructure that is characterized by periodic short fiber arrays. Each fiber is actually a parallelepiped particle.

(a) Rank-III laminates with linear and power-law components. The composites considered here are made of the following two materials:

$$
W^{(1)}(\mathbf{E})=\frac{1}{2} \sigma^{(1)} E^{2}, \quad W^{(2)}(\mathbf{E})=\frac{1}{2} \sigma^{(2)} E^{2}+\frac{1}{n+1} \omega^{(2)} E^{n+1},
$$

where $\sigma^{(1)}, \sigma^{(2)}, \omega^{(2)}, n(>1)$ are material constants. Nonlinear H-S bounds for these two-phase composites can be derived directly by substituting the potentials $W^{(1)}$ and $W^{(2)}$ into Eq. (87). This leads to the following optimization procedure:

$$
W_{\mathrm{HS}}(\overline{\mathbf{E}})=\frac{1}{2} \max _{\sigma_{0}^{(2)}>0}\left[\left(\bar{\sigma}_{0}\right)_{i} \bar{E}^{2}-\frac{n-1}{n+1} c^{(2)}\left(\sigma_{0}^{(2)}-\sigma^{(2)}\right)^{(n+1) /(n-1)}\left(\omega^{(2)}\right)^{-2 /(n-1)}\right] .
$$

When $\left(\bar{\sigma}_{0}\right)_{i}$ is defined by

$$
\left(\bar{\sigma}_{0}\right)_{i}=\sigma_{0}^{(1)}+\frac{3 c^{(2)} \sigma_{0}^{(1)}\left(\sigma_{0}^{(2)}-\sigma_{0}^{(1)}\right)}{c^{(1)} \sigma_{0}^{(2)}+\left(3-c^{(2)}\right) \sigma_{0}^{(1)}}, \quad \text { then } W_{\mathrm{HS} 1}=W_{\mathrm{HS}},
$$

and, when

$$
\left(\bar{\sigma}_{0}\right)_{i}=\sigma_{0}^{(2)}+\frac{3 c^{(1)} \sigma_{0}^{(2)}\left(\sigma_{0}^{(1)}-\sigma_{0}^{(2)}\right)}{c^{(2)} \sigma_{0}^{(1)}+\left(3-c^{(1)}\right) \sigma_{0}^{(2)}} \text {, then } W_{\mathrm{HS} 2}=W_{\mathrm{HS}} .
$$

Here $\sigma_{0}^{(1)}=\sigma^{(1)}$, and $c^{(1)}$ and $c^{(2)}$ are the volume fractions of the phases characterized by $W^{(1)}$ and $W^{(2)}$, respectively. The nonlinear H-S lower bound and the H-S estimate to the upper bound are given by

$$
W_{\mathrm{HS}-}=\min \left(W_{\mathrm{HS} 1}, W_{\mathrm{HS}_{2}}\right), \quad W_{\mathrm{HS}+}=\max \left(W_{\mathrm{HS} 1}, W_{\mathrm{HS}_{2}}\right) .
$$

In order to use (26) to calculate the effective energy function of this rank-III, three-dimensional laminate, we need to first obtain the effective comparison energy function $\widetilde{W}_{0}$. The effective conductivity of this linear comparison material has been 
derived in Appendix A, which is $\bar{\sigma}_{0}^{I I I}$ in (82) under the isotropic conditions in (81). Thus, the linear comparison energy function is

$$
\widetilde{W}_{0}(\overline{\mathbf{E}})=\frac{1}{2} \bar{\sigma}_{0}^{I I I} \bar{E}^{2} \text {. }
$$

Two nonlinear laminates are constructed. The first has a nonlinear matrix with linear inclusions. The second has a linear matrix with nonlinear inclusions.

Consider the laminate with linear inclusions (described by $W^{(1)}$ ) and nonlinear matrix (described by $W^{(2)}$ ). This laminate has three phases characterized by the nonlinear energy function $W^{(2)}$. By substituting $W^{(1)}$ and $W^{(2)}$ into (23) and (26), we obtain $\sigma_{0}^{(1)}=\sigma^{(1)}$ and the effective energy function in the form of an optimization, viz.,

$$
\frac{\widetilde{W}(\overline{\mathbf{E}})}{W^{(1)}} \max _{\substack{\sigma_{0}^{(2)}>0 \\ 1>c^{I}, c^{I I}>c^{(1)}}}\left[\frac{\bar{\sigma}_{0}^{I I I}}{\sigma^{(1)}}-F \frac{n-1}{n+1}\left(\frac{\omega^{(2)} \bar{E}^{n-1}}{\sigma^{(1)}}\right)^{-2 /(n-1)}\right],
$$

with $F$ defined by

$$
\begin{aligned}
F= & c^{I I I} c^{I I}\left(1-c^{I}\right)\left(\frac{\sigma_{0}^{(2)}-\sigma^{(2)}}{\sigma^{(1)}}\right)^{(n+1) /(n-1)} \\
& +c^{I I I}\left(1-c^{I I}\right)\left(\frac{\sigma_{0}^{(3)}-\sigma^{(2)}}{\sigma^{(1)}}\right)^{(n+1) /(n-1)}+\left(1-c^{I I I}\right)\left(\frac{\sigma_{0}^{(4)}-\sigma^{(2)}}{\sigma^{(1)}}\right)^{(n+1) /(n-1)} .
\end{aligned}
$$

The above optimization is originally with respect to the comparison conductivities $\sigma_{0}^{(2)}, \sigma_{0}^{(3)}$, and $\sigma_{0}^{(4)}$ which correspond to the comparison conductivities of the three nonlinear phases. The relative volume fractions $c^{I}$ and $c^{I I}$ are determined by the two isotropic conditions (or constraints) in Eq. (81). However, it is mathematically convenient to do the optimization with respect to $\sigma_{0}^{(2)}, c^{I}$, and $c^{I I}$ and use the two constraints to determine $\sigma_{0}^{(3)}$ and $\sigma_{0}^{(4)}$, since $c^{I}$ and $c^{I I}$ are limited to a specific domain.

For the other laminate-which has a linear matrix (i.e., with three phases characterized by $W^{(1)}$ ) and nonlinear inclusions (described by $W^{(2)}$ ) - the effective energy function can also be obtained by substituting $W^{(1)}$ and $W^{(2)}$ into (23) and (26). This leads to $\sigma_{0}^{(2)}=\sigma_{0}^{(3)}=\sigma_{0}^{(4)}=\sigma^{(1)}$. The resulting nonlinear energy function is obtained by an optimization with respect to one variable, $\sigma_{0}^{(1)}$, and coincides with the nonlinear H-S bound, $W_{\mathrm{HS} 2}$, defined by Eq. (47) when $\left(\bar{\sigma}_{0}\right)_{i}$ is defined by (49).

(b) Laminates with pure power-law components. The composites considered here are composed of two pure power-law materials, both of which have the same nonlinear order but different nonlinear conductivities:

$$
W^{(1)}(\mathbf{E})=\frac{1}{n+1} \omega^{(1)} E^{n+1}, \quad W^{(2)}(\mathbf{E})=\frac{1}{n+1} \omega^{(2)} E^{n+1},
$$

where $\omega^{(1)}, \omega^{(2)}, n(>1)$ are material constants. The resulting isotropic effective 
energy functions have the same form as the phase materials $\left(W^{(1)}\right.$ and $\left.W^{(2)}\right)$, namely

$$
\widetilde{W}(\overline{\mathbf{E}})=\frac{1}{n+1} \widetilde{\omega} \bar{E}^{n+1},
$$

where $\widetilde{\omega}$ is the effective nonlinear conductivity [22]). Since this functional form for the effective energy is known, the effective properties are characterized by $\widetilde{\omega}$. Blumenfeld and Bergman [22] have studied these two-phase composites when $\omega^{(2)} / \omega^{(1)}$ $\sim 1$, and obtained a second-order upper bound for the effective conductivity $\widetilde{\omega}$.

Voigt and Reuss bounds can also be derived for this composite. Using the minimum energy principle $\left(\widetilde{W} \leq c^{(1)} W^{(1)}+c^{(2)} W^{(2)}\right)$, the Reuss upper bound can be obtained directly, viz.,

$$
\frac{\widetilde{\omega}}{\omega^{(1)}} \leq c^{(1)}+c^{(2)} \frac{\omega^{(2)}}{\omega^{(1)}},
$$

where $c^{(2)}$ and $c^{(1)}$ are volume fractions of phases with conductivity characterized by $W^{(1)}$ and $W^{(2)}$ respectively. The Voigt lower bound is derived from minimum complementary energy and is given by

$$
\frac{\widetilde{\omega}}{\omega^{(1)}} \geq\left[c^{(1)}+c^{(2)}\left(\frac{\omega^{(2)}}{\omega^{(1)}}\right)^{-1 / n}\right]^{-n} .
$$

The nonlinear H-S bounds are again derived using linear H-S bounds as the comparison material in Ponte Castañeda's variational principle as outlined in Appendix B. Substituting the potentials $W^{(1)}$ and $W^{(2)}$ into Eq. (87) leads to

$$
\begin{aligned}
W_{\mathrm{HS}}(\overline{\mathbf{E}}) & =\frac{1}{2} \max _{\sigma_{0}^{(1)}, \sigma_{0}^{(2)}>0}\left\{\left(\bar{\sigma}_{0}\right)_{i} \bar{E}^{2}\right. \\
& \left.-\frac{n-1}{n+1}\left[c^{(1)}\left(\sigma_{0}^{(1)}\right)^{(n+1) /(n-1)}\left(\omega^{(1)}\right)^{-2 /(n-1)}+c^{(2)}\left(\sigma_{0}^{(2)}\right)^{(n+1) /(n-1)}\left(\omega^{(2)}\right)^{-2 /(n-1)}\right]\right\},
\end{aligned}
$$

where $\left(\bar{\sigma}_{0}\right)_{i}$ is given either by (48) or by (49). The nonlinear H-S lower bound and estimate to the upper bound are again determined by $(50)$.

Since the microstructure of the rank-III laminate studied here is the same as that in the last subsection, the effective energy of the linear comparison material is again $\widetilde{W}_{0}(\overline{\mathbf{E}})=(1 / 2) \bar{\sigma}_{0}^{I I I} \bar{E}^{2}$. Two different isotropic laminates are considered here. Both are required to meet the isotropy conditions in Eq. (81).

The first laminate (Laminate 1) has inclusions characterized by $W^{(1)}$ and matrix characterized by $W^{(2)}$. The application of Eqs. (23) and (26) leads to the nonlinear effective conductivity

$$
\frac{\widetilde{\omega}}{\omega^{(1)}}=\max _{\substack{\sigma_{0}^{(1)},,_{0}^{(2)}>0 \\ 1>c^{I}, c^{I I}>c^{(1)}}}\left[\frac{n+1}{2} \frac{\bar{\sigma}_{0}^{I I I}}{\omega^{(1)}} \bar{E}^{1-n}-\frac{n-1}{2} F \bar{E}^{1+n}\right],
$$


with $F$ defined by

$$
\begin{aligned}
F=c^{(1)}\left(\frac{\sigma_{0}^{(1)}}{\omega^{(1)}}\right)^{(n+1) /(n-1)}+\left[c^{I I I} c^{I I}\left(1-c^{I}\right)\left(\frac{\sigma_{0}^{(2)}}{\omega^{(1)}}\right)^{(n+1) /(n-1)}\right. \\
\left.+c^{I I I}\left(1-c^{I I}\right)\left(\frac{\sigma_{0}^{(3)}}{\omega^{(1)}}\right)^{(n+1) /(n-1)}+\left(1-c^{I}\right)\left(\frac{\sigma_{0}^{(4)}}{\omega^{(1)}}\right)^{(n+1) /(n-1)}\right]\left(\frac{\omega^{(2)}}{\omega^{(1)}}\right)^{-2 /(n-1)},
\end{aligned}
$$

where $c^{I I I} c^{I I} c^{I}=c^{(1)}$, and $c^{(1)}$ is the volume concentration of $W^{(1)}$. This laminate has nonlinear materials in all of its four phases. Thus, the effective energy function is an optimization with respect to the properties of the four phases of the linear comparison material (represented by conductivities $\sigma_{0}^{(1)}, \sigma_{0}^{(2)}, \sigma_{0}^{(3)}$, and $\sigma_{0}^{(4)}$ ). The relative volume fractions $c^{I}$ and $c^{I I}$ are determined by the two isotropic conditions in Eq. (81). Again, since $c^{I}$ and $c^{I I}$ are in a specific domain $\left(1>c^{I}, c^{I I}>c^{(1)}\right)$, it is mathematically convenient to optimize with respect to $c^{I}$ and $c^{I I}$ instead of $\sigma_{0}^{(3)}$ and $\sigma_{0}^{(4)}$. The two isotropic conditions in Eq. (81) are then used to determine $\sigma_{0}^{(3)}$ and $\sigma_{0}^{(4)}$.

The second laminate (Laminate 2) has matrix and inclusions characterized by $W^{(1)}$ and $W^{(2)}$ respectively. The same procedure as that described above leads to an effective conductivity in a form similar to (59), namely

$$
\frac{\widetilde{\omega}}{\omega^{(1)}}=\max _{\substack{\sigma_{0}^{(1)}, \sigma_{0}^{(2)}>0 \\ 1>c^{I}, c^{I I}>c^{(2)}}}\left[\frac{n+1}{2} \frac{\bar{\sigma}_{0}^{I I I}}{\omega^{(1)}} \bar{E}^{1-n}-\frac{n-1}{2} F \bar{E}^{1+n}\right],
$$

but with $F$ defined by

$$
\begin{aligned}
F= & c^{(2)}\left(\frac{\sigma_{0}^{(1)}}{\omega^{(1)}}\right)^{(n+1) /(n-1)}\left(\frac{\omega^{(2)}}{\omega^{(1)}}\right)^{-2 /(n-1)}+c^{I I I} c^{I I}\left(1-c^{I}\right)\left(\frac{\sigma_{0}^{(2)}}{\omega^{(1)}}\right)^{(n+1) /(n-1)} \\
& +c^{I I I}\left(1-c^{I I}\right)\left(\frac{\sigma_{0}^{(3)}}{\omega^{(1)}}\right)^{(n+1) /(n-1)}+\left(1-c^{I}\right)\left(\frac{\sigma_{0}^{(4)}}{\omega^{(1)}}\right)^{(n+1) /(n-1)},
\end{aligned}
$$

where $c^{I I I} c^{I I} c^{I}=c^{(2)}$, and $c^{(2)}$ is the volume concentration of $W^{(2)}$. The calculation of the effective conductivity $\widetilde{\omega}$ of the above two laminates is accomplished through an iterative procedure. For initial values of the optimization variables $\left(\sigma_{0}^{(1)}, \sigma_{0}^{(2)}, c^{I}\right.$, and $\left.c^{I I}\right)$, the isotropy conditions of Eq. (81) are solved to get $\sigma_{0}^{(3)}$, $\sigma_{0}^{(4)}$. They are substituted in the optimization equations to obtain new values of the optimization variables. This procedure continues to reach a convergent solution for the effective conductivity parameter $\widetilde{\omega}$.

V. Results. The objective of this work is to obtain the effective conductivity of nonlinear isotropic laminate composite materials. This conductivity, characterized by the effective energy $\widetilde{W}$, is dependent on the magnitude of the applied electric field $\bar{E}$. 
In Figs. 2 and 3, phase 1 is linear, characterized by the potential $W^{(1)}$ in (27) and has concentration $c^{(1)}$. Phase 2 is nonlinear, with potential $W^{(2)}$ in (27) and concentration $c^{(2)}\left(=1-c^{(1)}\right)$. In order to present the data in the most compact and general form we will plot the effective energy of the laminate composite nondimensionalized by the effective energy of its linear phase, namely $\widetilde{W} / W^{(1)}$, versus the normalized electric field intensity. The normalization, which appears earlier in Eqs. (33) and (52), is chosen such that the results hold for all nonlinear materials $\left(W^{(2)}\right)$ of a given nonlinear order $n$. We therefore plot $\widetilde{W} / W^{(1)}$ versus $\omega^{(2)} \bar{E}^{n-1} / \sigma^{(1)}$, where $\omega^{(2)}$ and $n$ characterize phase 2 and $\sigma^{(1)}$ is the conductivity of phase 1; see (27).

(a) Two-dimensional laminates. Figures $2(a),(b),(c)$, and (d) show results for two-dimensional laminates with phase 1 linear and phase 2 nonlinear. We confine ourselves to the discussion of one typical material of moderate nonlinear order $(n=$ 3 ) and one of high nonlinear order $(n=10)$. In each of these figures the effective conductivities of the rank-II and rank-III laminates are presented and compared with the nonlinear $\mathrm{H}-\mathrm{S}$ bounds. Recall that the nonlinear $\mathrm{H}-\mathrm{S}$ lower bound is a strict lower bound while the nonlinear H-S upper bound is an estimate to the true bound. Results for five discrete concentrations of the linear material $c^{(1)}=0.0,0.1,0.5,0.8,1.0$ are shown.

Figures 2(a) and 2(b) (see p. 452) examine the effective conductivity of a laminate at a small applied electric field. For an exclusively linear material, $c^{(1)}=1.0$ and $\widetilde{W} / W^{(1)}=1$ for all $\bar{E}$, while for an exclusively power-law material, $c^{(1)}=0.0$ and $\widetilde{W}$ is proportional to $\omega^{(2)} \bar{E}^{n-1} / \sigma^{(1)}$ as required by the nondimensionalization.

For each concentration $c^{(1)}$, two materials are possible. The first has linear inclusions and nonlinear matrix, while the second has nonlinear inclusions in a linear matrix. Since the applied electric field is small $\left(0.0<\omega^{(2)} \bar{E}^{n-1} / \sigma^{(1)}<1.0\right)$ the linear material is more conductive and the composite with linear matrix has higher effective conductivity.

In both figures the potentials representing the nonlinear $\mathrm{H}-\mathrm{S}$ bounds, $W_{\mathrm{HS}-}$ and $W_{\mathrm{HS}+}$, are plotted for each concentration of the linear material. Both the rank-II and rank-III materials in which the matrix material is linear (the more conductive arrangement in this range of applied field) coincide with the nonlinear $\mathrm{H}-\mathrm{S}$ estimate to the upper bound $W_{\mathrm{HS}+}$ defined by (28) with (30). Since these results are identi$\mathrm{cal}$, only the estimate for nonlinear H-S upper bound is plotted. Even though linear counterparts of both these rank-II and rank-III laminated microstructures attain the linear H-S upper bound, we cannot say that they are also extremal nonlinear composite microstructures. This is because we have only an estimate of the upper bound to which the conductivity of these nonlinear materials corresponds.

For materials with nonlinear matrix material, the effective conductivities for both the rank-II and rank-III laminates are very close to the $\mathrm{H}-\mathrm{S}$ lower bound, $W_{\mathrm{HS}-}$. (The rank-III laminate is closer than the rank-II laminate.) The corresponding linear laminated microstructures (with the more strongly conducting material as inclusions) attain the linear H-S lower bound and are therefore extremal materials. Although we 


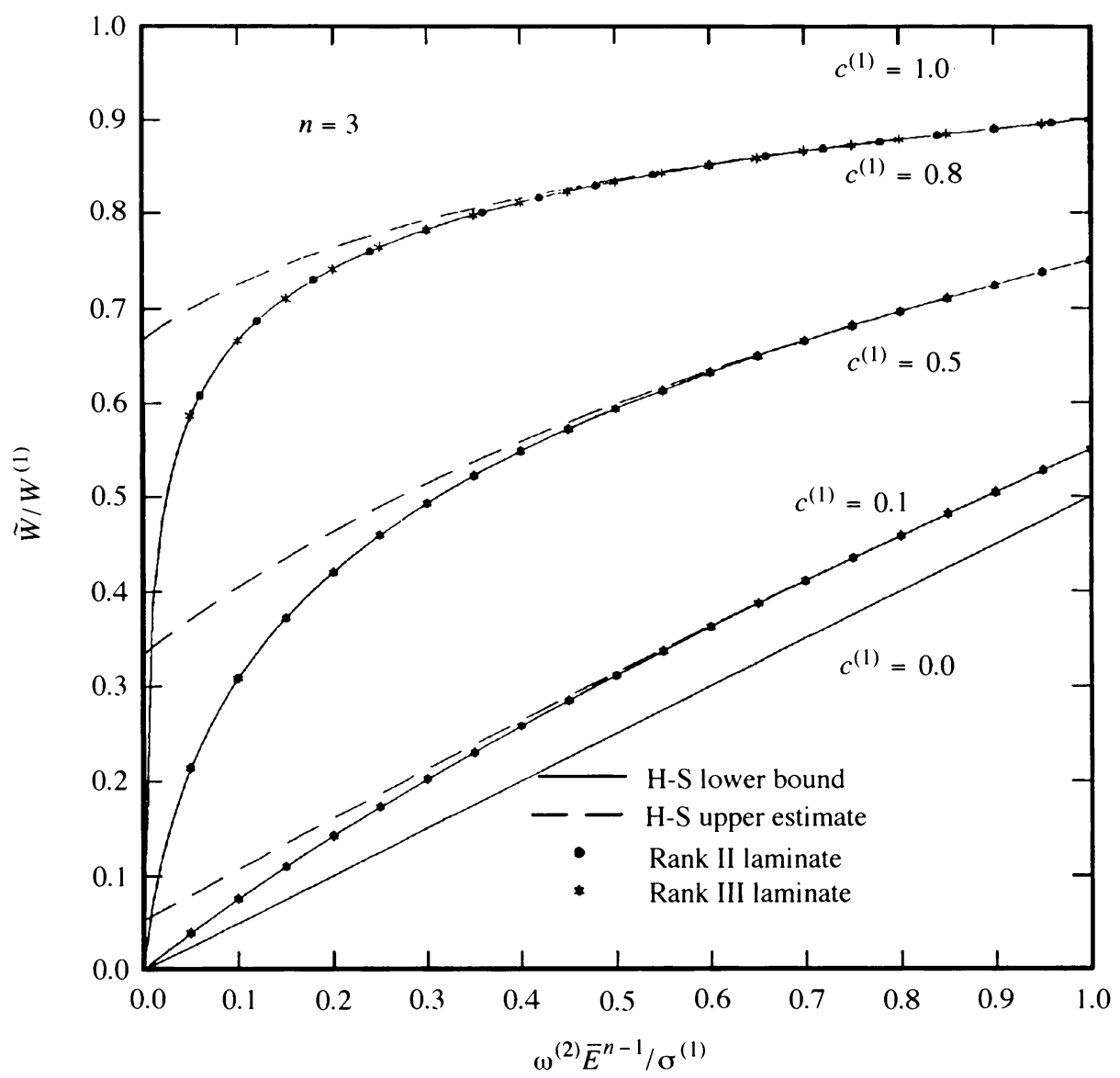

FIG. 2(a). The effective energy of nonlinear, isotropic, two-dimensional, rank-II and rank-III laminates as a function of average electric field, $\bar{E}$, and the nonlinear $H-S$ lower-bound and upper-bound estimate. The two-phase laminates are composed of a power law and a linear phase, where $c^{(1)}$ denotes the volume fraction of the linear phase. The laminates with linear inclusions coincide with the $\mathrm{H}-\mathrm{S}$ bound $W_{\mathrm{HS} 2}$; thus, only $W_{\mathrm{HS} 2}$ is plotted. Weak nonlinearity $(n=3)$ and small electric field. Here, the rank-II laminates with nonlinear matrix almost coincide with the $\mathrm{H}-\mathrm{S}$ nonlinear lower bound $W_{\mathrm{HS} 1}$.

cannot assert that the nonlinear composites constructed here are extremal materials, the proximity of their effective responses to the nonlinear H-S lower bound indicates that this bound is very close to the true lower bound.

Figures 2(c) and 2(d) (see pp. 453, 454) examine the same materials in the same manner as Figs. 2(a) and 2(b), but for a larger range of electric field. The significant feature of Figs. 2(c) and 2(d) is that there is a transition region around $\omega^{(2)} \bar{E}^{n-1} / \sigma^{(1)} \sim 1$ where the effective property $\widetilde{W}$ for both laminated materials and both the nonlinear H-S lower-bound and upper-bound estimates are identical to within five significant figures. This region is smaller when the concentration of linear 


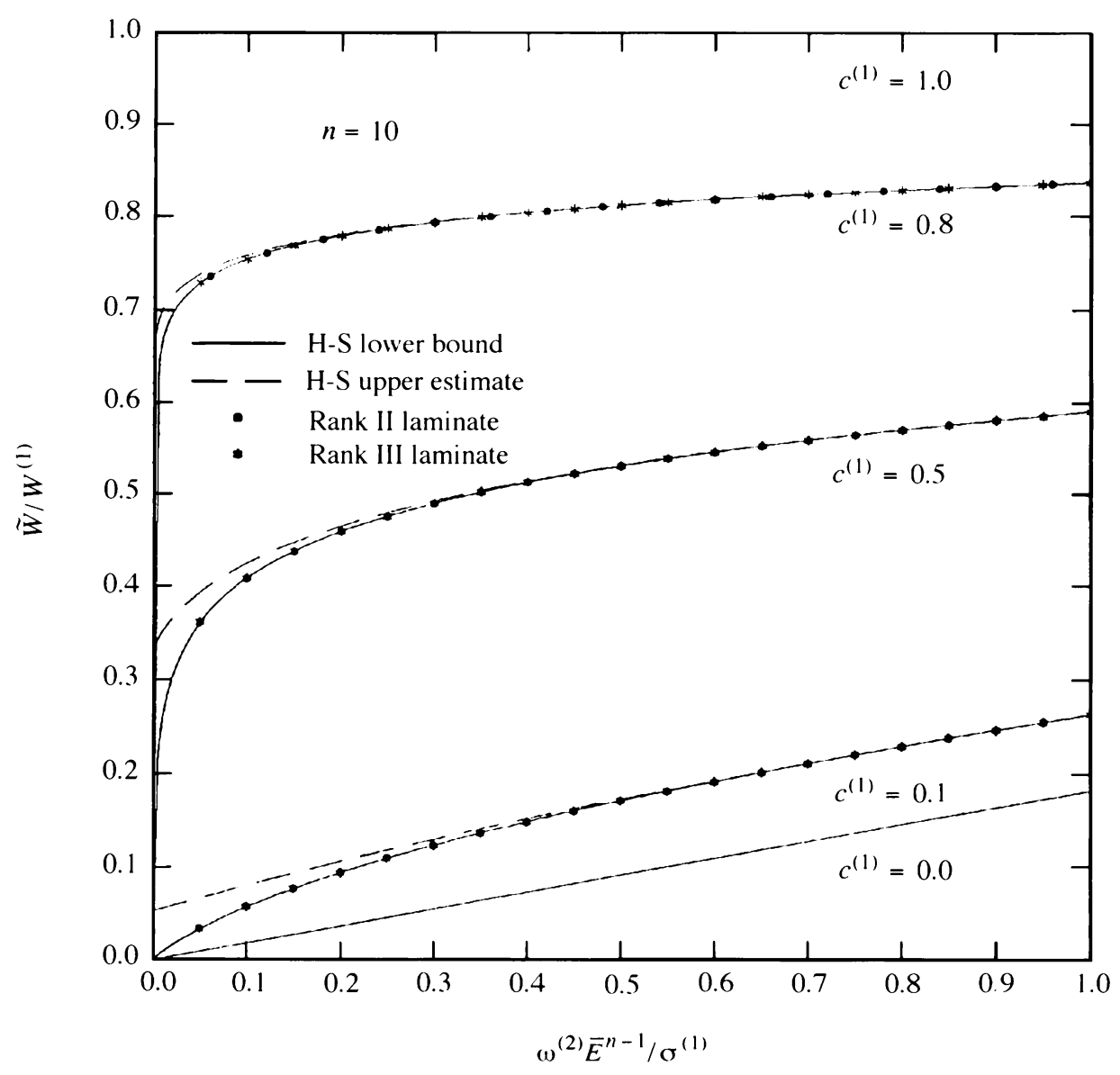

FIG. 2(b). Strong nonlinearity $(n=10)$ and small electric field. The rank-II laminates with nonlinear matrix again almost coincide with nonlinear $\mathrm{H}-\mathrm{S}$ lower bound $W_{\mathrm{HS} 1}$.

phase is small $\left(c^{(1)}\right.$ is small) than for high-volume fraction of linear material. In this region of applied electric field magnitude both nonlinear and linear phases respond in a similar manner so that all the two-phase composites composed of both linear and power-law constituents have almost identical conductive behavior. Both the laminates and the H-S bounds provide an accurate estimate for the effective conductive behavior.

To the right of this region, the electric field intensity is large enough to ensure that the nonlinear phase is more conductive than the linear phase. Also, the nonlinear $\mathrm{H}-\mathrm{S}$ bound (28) with (29) and (30) results in $W_{\mathrm{HS} 2}<W_{\mathrm{HS} 1}$, i.e., $W_{\mathrm{HS}-}=W_{\mathrm{HS}_{2}}$ is a strict lower bound. We have already shown that the laminate with linear matrix coincides with $W_{\mathrm{HS} 2}$ for the entire range of electric field. Thus, at large electric field $\left(\omega^{(2)} \bar{E}^{n-1} / \sigma^{(1)}>1\right)$, the sequentially laminated materials provide a series of extremal microstructures achieving the nonlinear H-S lower bound. This in turn proves that the nonlinear H-S bound is a true lower bound at large electric field. 


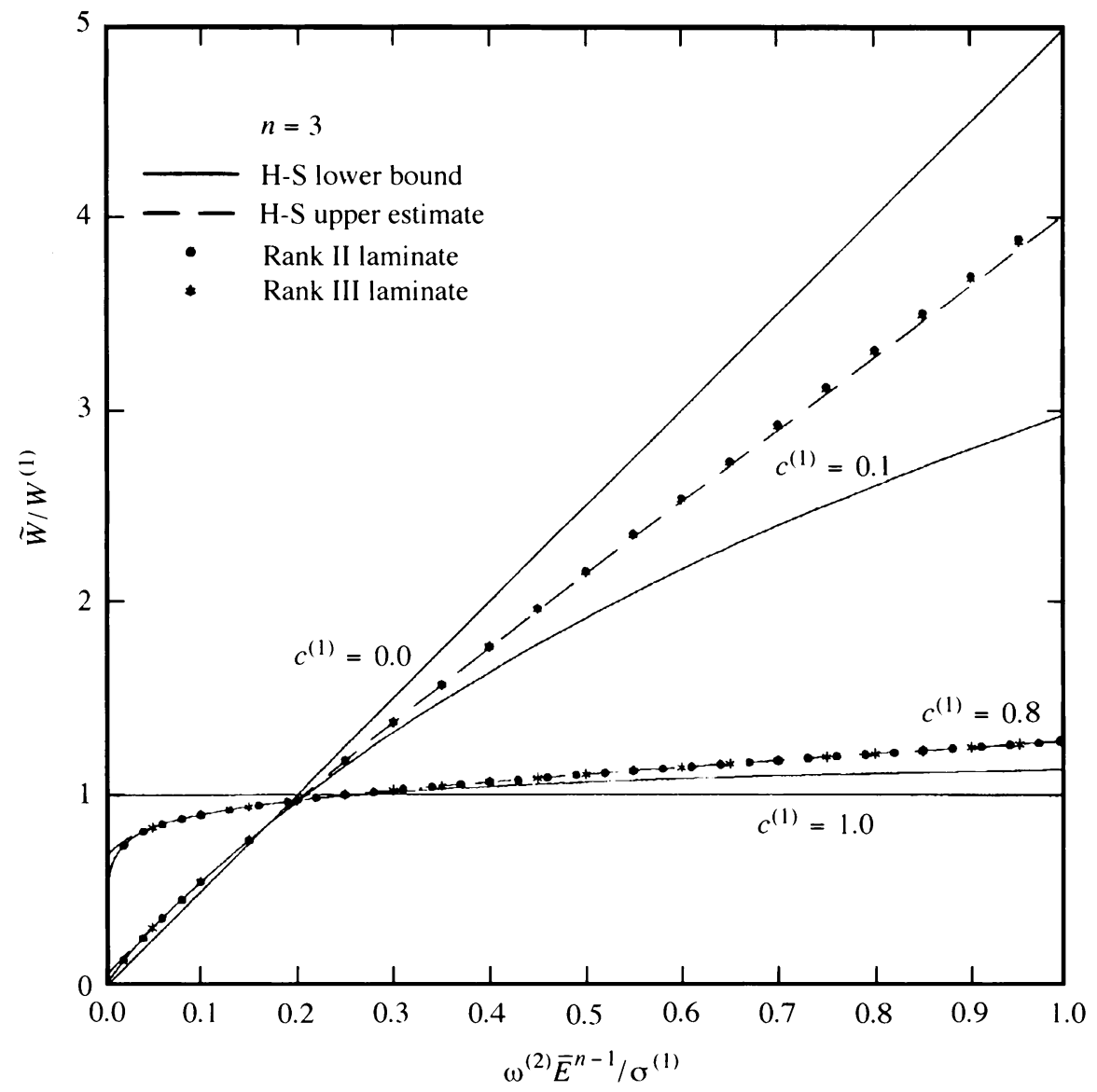

Fig. 2(c). Weak nonlinearity $(n=3)$ and a large range for the applied electric field.

Both rank-II and rank-III laminates lie above the estimate for the upper H-S bound, $W_{\mathrm{HS}+}$. The rank-III laminated material is closer to the estimate $W_{\mathrm{HS}+}$ than the rankII material. This confirms the fact that $W_{\mathrm{HS}+}$ is not a true upper bound.

In comparing Fig. 2(a) with 2(b), and Fig. 2(c) with 2(d), it is evident that the range of possible material behavior for a given electric field is larger for the material with lower nonlinear order $n$.

(b) Three-dimensional laminates. Figures 3(a) and 3(b) (see pp. 455, 456) show the effective conductivity for three-dimensional nonlinear laminate composites. In this case, phase 1 is linear and phase 2 is linear plus power-law (see Eq. (22)) with the linear coefficient chosen such that $\sigma^{(2)} / \sigma^{(1)}=2 .>1$, which guarantees that the nonlinear material is always more conductive, regardless of the electric field intensity. This also means that the nonlinear H-S lower bound is always given by (47) and (49). The estimate to the nonlinear upper bound is given by (47) and (48).

Two nonlinear materials, one with moderate nonlinearity $(n=3)$ and the other with strong nonlinearity $(n=10)$ are again studied. Independent of concentration, 


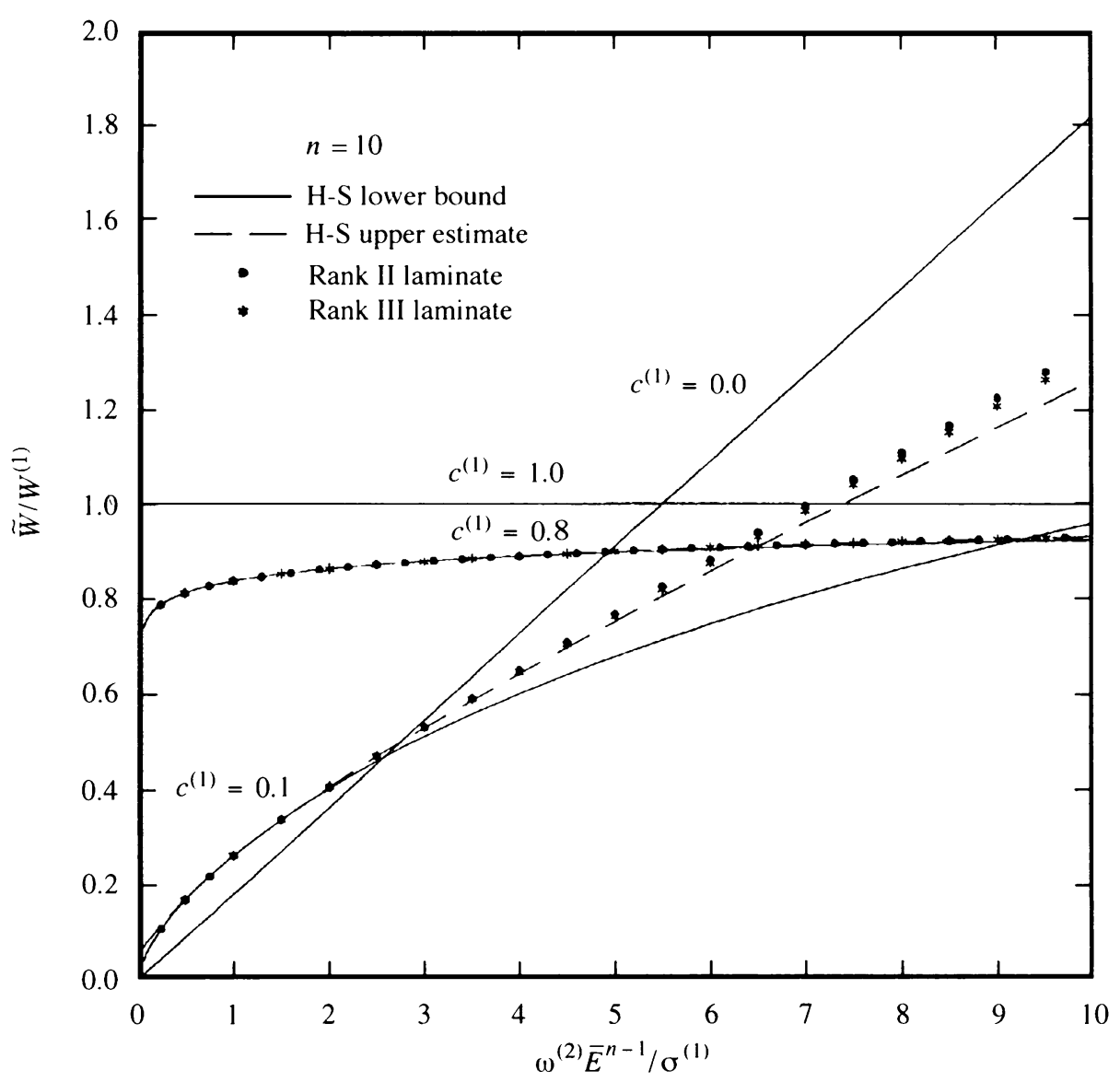

Fig. 2(d). Strong nonlinearity $(n=10)$ and a large range for the electric field.

the laminate with linear matrix has an effective conductivity (as mentioned earlier) given by $\widetilde{W}=W_{\mathrm{HS} 2}=W_{\mathrm{HS} \text { - }}$ and is therefore an extremal microstructure that achieves the nonlinear $\mathrm{H}-\mathrm{S}$ lower bound.

The estimate for the upper bound, $W_{\mathrm{HS}+}$, is plotted as a dashed line for each concentration $c^{(1)}$. The laminates with nonlinear matrix have effective potentials slightly above these dashed lines when $n=3$. For the strongly nonlinear matrix $(n=10)$, the difference between the laminate and the estimate to the nonlinear upper bound is slightly larger than that for $n=3$ as $\bar{E}$ becomes larger. Although the effective potentials for the nonlinear upper bound estimate, $W_{\mathrm{HS}_{+}}$, and the laminates, $\widetilde{W}$, are close to each other, we cannot conclude that $W_{\mathrm{HS}+}$ is a true bound nor that these laminate composites are extremal materials.

(c) Three-dimensional laminates with two power-law phases. Figures $4(\mathrm{a})$ and 4(b) (see pp. 457, 458) show the effective electrical conductivities of laminate materials with the pure power-law constituents discussed earlier (54). We again consider materials of nonlinear order $n=3$ or $n=10$. In this case the volume fraction 


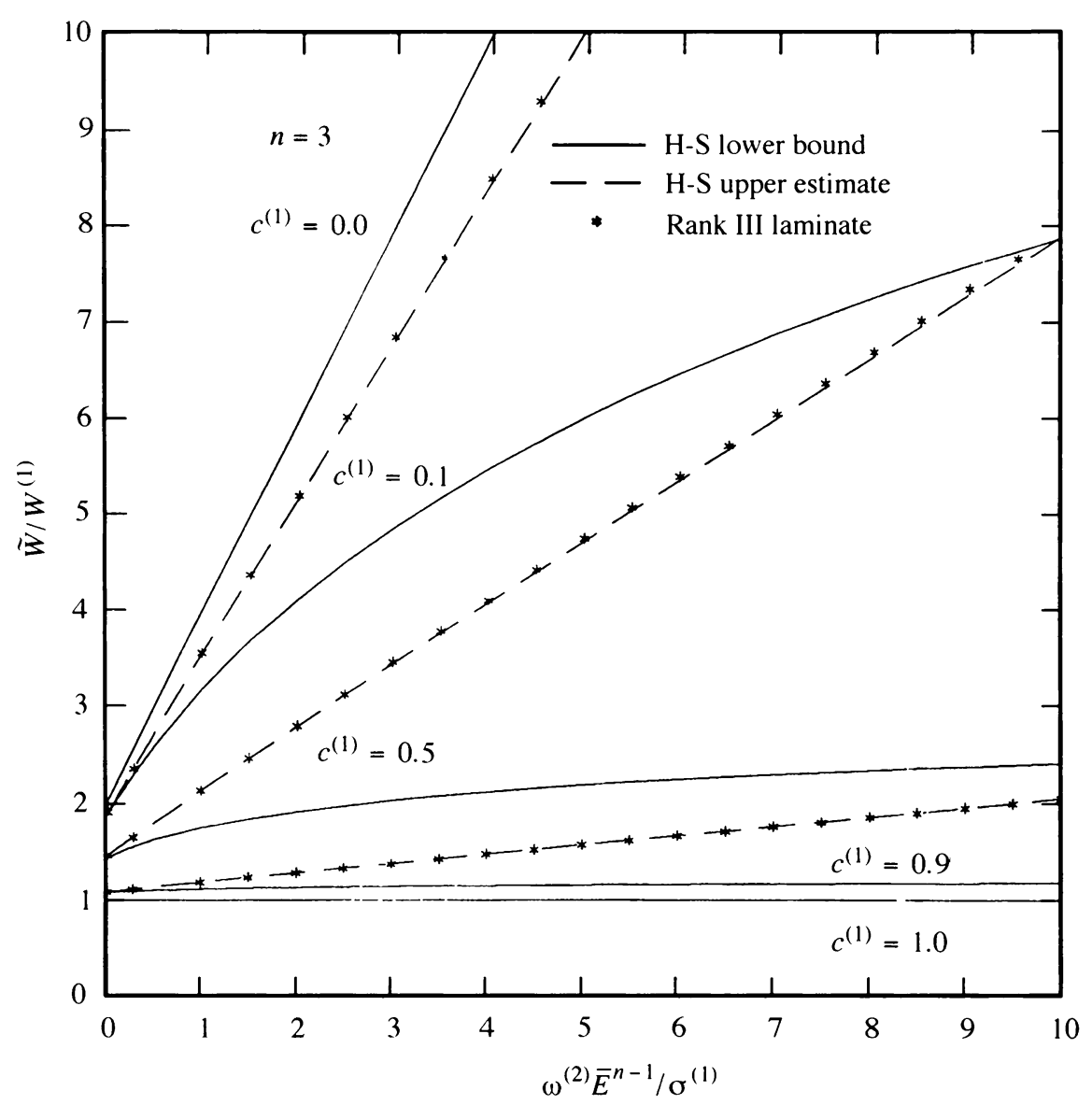

Fig. 3(a). The effective electrical conductivity of nonlinear, isotropic, three-dimensional rank-III laminates (composed of linear plus powerlaw constituents) as a function of the nondimensionalized applied electric field. Weak nonlinearity $(n=3)$.

of the less conductive material, characterized by $W^{(2)}$, is $c^{(2)}$. Since the form of the effective potential $\widetilde{W}$ is known, $\widetilde{\omega}$ characterizes this potential (see (55)). Also, since both phases of the composite have the same nonlinear order $n$, the effective conductivity is independent of the applied electric field magnitude $\bar{E}$. We therefore plot the nondimensionalized effective conductivity, $\widetilde{\omega} / \omega^{(1)}$, versus the ratio of the conductivity of the less conductive phase to the more conductive phase, $\omega^{(2)} / \omega^{(1)}$.

Neither the laminates with the less conductive material as matrix (Laminate 1) nor as inclusions (Laminate 2) attain the nonlinear H-S bounds. Both these rank-III laminates are plotted as stars in Figs. $4(a)$ and $4(b)$ for the concentration $c^{(2)}=0.5$.

Although Laminate 1 is not formally an extremal material, the effective nonlinear conductivity of Laminate 1 (with the more conductive inclusions) is so close to the H-S lower bound (58) that it effectively shows that the H-S lower bound is a tight bound and that this material is, for practical purposes, an extremal material. 


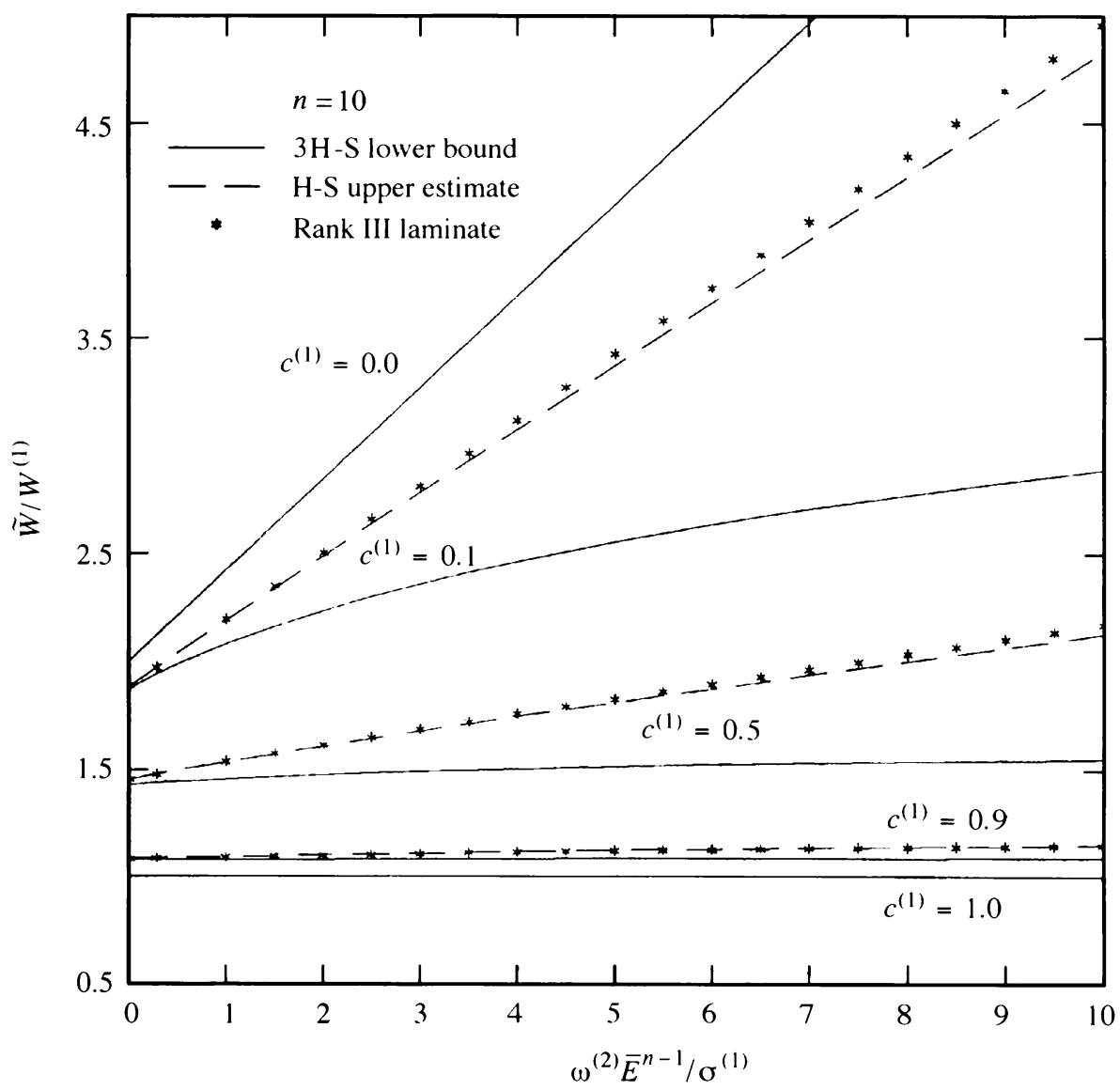

FIG. 3(b). The effective electrical conductivity of nonlinear, isotropic, three-dimensional rank-III laminates (composed of linear plus powerlaw constituents) as a function of the nondimensionalized applied electric field. Strong nonlinearity $(n=10)$.

Laminate 2 has effective conductivity slightly above the estimate for the nonlinear upper bound (58). This shows again that the laminate results are closer to the true upper bound than the H-S upper-bound estimate.

In order to compare the laminate results with other results, the Voigt upper and Reuss lower bounds are plotted in both Figs. 4(a) and 4(b) as indicated. Also plotted is the Blumenfeld and Bergman (B-B) second-order upper bound [22], which lies between the Voigt upper bound and the rank-III laminate. When the component conductivities are similar, $\omega^{(2)} / \omega^{(1)} \sim 1$, the B-B upper bound is larger than but very close to Laminate 2. This shows that the B-B upper bound is a better estimate to the optimal upper bound than the Voigt bound. Actually, when $\omega^{(2)} / \omega^{(1)}>0.8$, all the calculations are so close to each other that each of them well represents the effective conductivity of a general two-phase composite with pure power-law constituents. When the constituent conductivities are very different $\left(\omega^{(2)} / \omega^{(1)}\right.$ is small), the dif- 


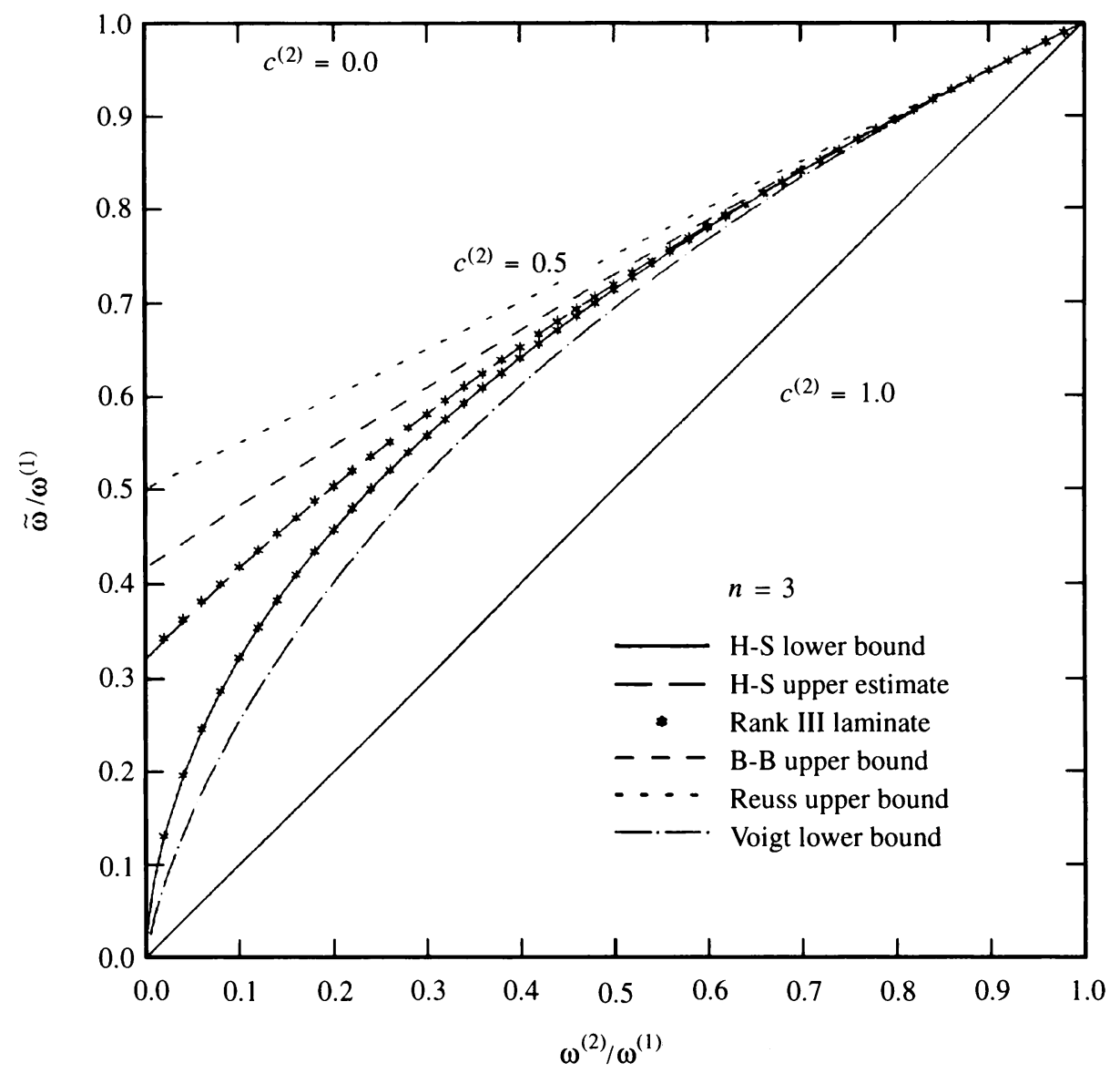

Fig. 4(a). The effective electrical conductivities of nonlinear, isotropic, three-dimensional rank-III laminates (which are made of two pure power-law materials) as a function of the ratio of the nonlinear conductivities of the two different constituent materials. Here $c^{(2)}$ denotes the volume fraction of the less conductive material $W^{(2)}$. Weak nonlinearity $(n=3)$.

ference between the B-B upper bound and Laminate 2 increases. But, the laminate results are still very close to the H-S upper-bound estimate.

When $\omega^{(2)}=0\left(\omega^{(2)} / \omega^{(1)}=0\right)$, in Figs. $4(a)$ and $4(b)$ the effective conductivity of laminates with $W^{(2)}$ as matrix is zero $(\widetilde{\omega}=0)$, since $W^{(2)}$ is a perfect insulator; inclusions are perfect insulators. When $c^{(2)}=1$, the entire composite is characterized by $W^{(2)}$ and all the results (H-S bounds and laminate materials) collapse onto the diagonal line shown. Also, when $c^{(2)}=0$, the entire composite is characterized by $W^{(1)}$ and all the results (H-S bounds and laminate materials) collapse onto the horizontal line $\widetilde{\omega}=\omega^{(1)}$. 


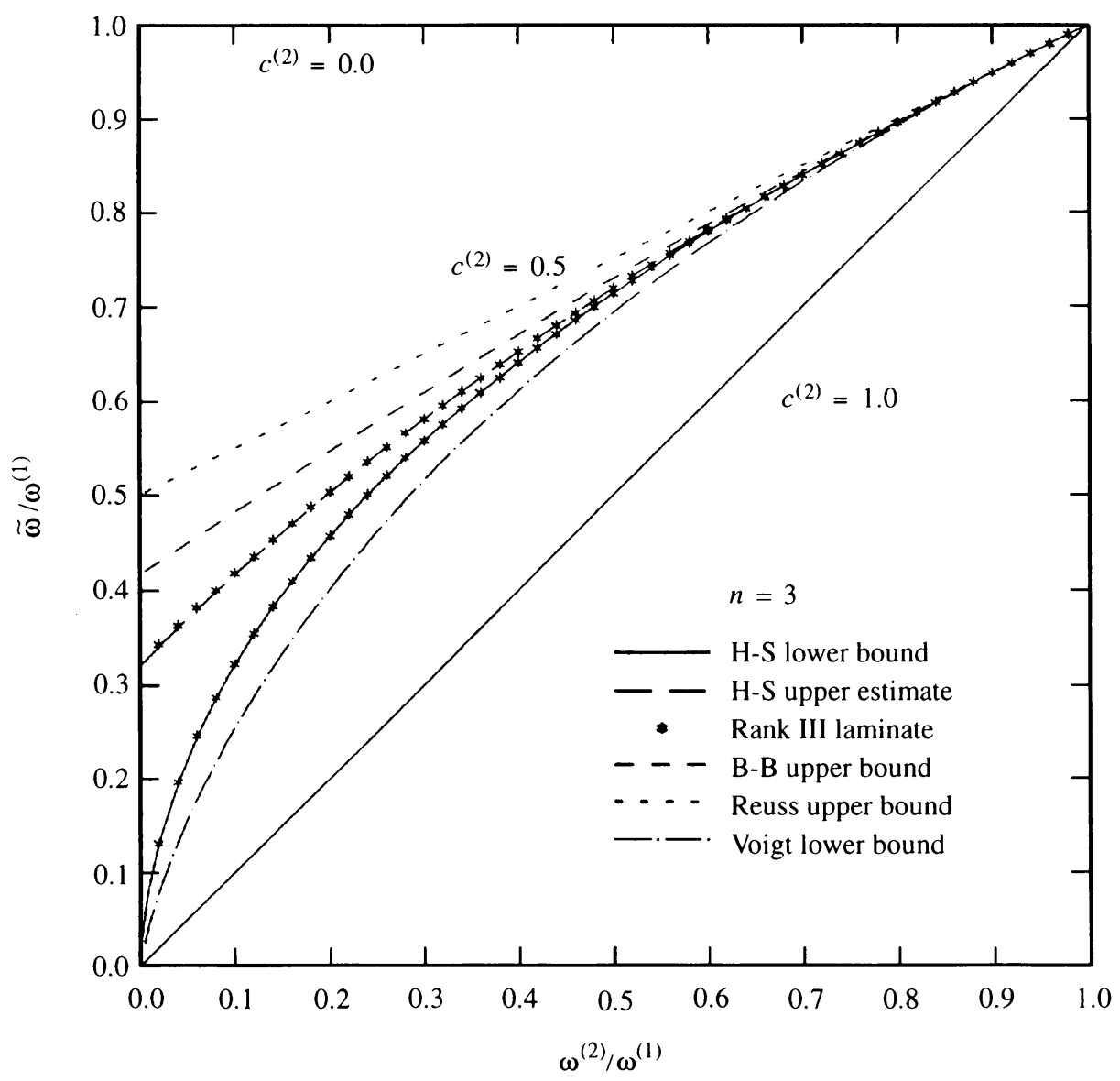

Fig. 4(b). The effective electrical conductivities of nonlinear, isotropic, three-dimensional rank-III laminates (which are made of two pure power-law materials) as a function of the ratio of the nonlinear conductivities of the two different constituent materials. Here $c^{(2)}$ denotes the volume fraction of the less conductive material $W^{(2)}$. Strong nonlinearity $(n=10)$.

VI. Conclusions. Using the solutions for laminate composites made of linear materials with concentrations chosen to ensure an isotropic material response, the variational principle due to Ponte Castañeda [1] can be used to find the exact effective electrical conductivity for isotropic two-phase nonlinear laminated materials. When the more strongly conducting of the two phases (which may depend on the applied electric field intensity $\bar{E}$ ) comprises the inclusions, these composites are either extremal materials (i.e., they attain the nonlinear H-S bounds) or they are very close to extremal microstructures. On the other hand, when the less conductive material forms the inclusions, their effective conductivity is closer to the true upper bound than the H-S estimate of the nonlinear upper bound.

For linear materials, all the two-phase, isotropic, sequentially laminated materials of any rank attain the corresponding linear H-S bounds. They are therefore all extremal linear materials. For nonlinear materials, each two-phase, isotropic material 
of different rank (for a given ratio of concentrations) has a slightly different electrical conductivity. When the inclusions are more conductive, the higher the rank, the closer is the effective conductivity to the H-S lower bound. It is expected that the sequentially laminated materials may converge to the optimal lower bound as the rank of the laminates increases.

On the other hand, when the less conductive material forms the inclusions, the lowest rank, isotropic laminate (i.e., the simplest microstructure) is the material closest to the true nonlinear upper bound.

The fact that the effective properties for these nonlinear laminated materials are obtained exactly and that the conductivities are so close to the nonlinear H-S lower bound also means that this bound is a tight bound. These exact solutions provide powerful tools in testing other nonlinear bounds as shown in the case of the B-B upper bound. They also provide an insight to the properties of nonlinear heterogeneous materials.

These laminates provide a simple way of obtaining effective properties of nonlinear electrical and thermal conductors and dielectric and magnetic materials over the entire range of inclusion concentration. In principle, this method could also be used to determine the effective properties of nonlinear elastic materials. However, since the elasticity tensor is fourth rank, the construction of an isotropic laminate linear comparison material is prohibitively complex.

Acknowledgments. The authors would like to thank Professor Ponte Castañeda for his valuable comments and suggestions. Both authors would like to acknowledge the support of Johns Hopkins University. Additional support for GL was provided by the University of Pennsylvania.

\section{Appendix A. Linear laminated materials.}

(a) Two-dimensional laminates. Consider electrical conductivity in the $\left(x_{1}, x_{2}\right)$ plane only. A rank-I laminate is shown in Fig. 1(a). Both composite phases are isotropic with conductivities $\sigma_{0}^{(1)}$ and $\sigma_{0}^{(2)}$ and are laminated in the $x_{2}$ direction. The effective conductivity tensor $\overline{\boldsymbol{\sigma}}^{I}$ of this laminate, obtained from (17), is

$$
\left[\bar{\sigma}^{I}\right]=\left[\begin{array}{cc}
\bar{\sigma}_{11}^{I} & 0 \\
0 & \bar{\sigma}_{22}^{I}
\end{array}\right]
$$

with

$$
\bar{\sigma}_{11}^{I}=c^{I} \sigma_{0}^{(1)}+\left(1-c^{I}\right) \sigma_{0}^{(2)}, \quad \bar{\sigma}_{22}^{I}=\left(c^{I} / \sigma_{0}^{(1)}+\left(1-c^{I}\right) / \sigma_{0}^{(2)}\right)^{-1},
$$

where $c^{I}$ is the relative volume fraction of phase 1 in the rank-I laminate, and $\bar{\sigma}_{11}^{I}$ and $\bar{\sigma}_{22}^{I}$ are the electrical conductivities in the $x_{1}$ and $x_{2}$ directions, respectively.

We examine two kinds of rank-II laminates, both of which are constructed using the rank-I laminate in Fig. 1(a) as one component and another isotropic material (with electrical conductivity $\sigma_{0}^{(3)}$ ) as the other component. Each of the rank-II laminates is laminated in a different direction $\mathbf{n}$. The relative volume fraction of the rank-I laminate is $c^{I I}$ while the isotropic material has volume fraction $1-c^{I I}$. Figure 1(b) shows an orthogonal rank-II laminate composed by laminating in the $x_{1}$ 
direction $\left(\mathbf{n}=x_{1}\right)$. The effective electric conductivity $\overline{\boldsymbol{\sigma}}^{I I}$ (two-dimensional), as given by (17), is also diagonal and may be represented by the matrix

with

$$
\left[\overline{\boldsymbol{\sigma}}^{I I}\right]=\left[\begin{array}{cc}
\bar{\sigma}_{11}^{I I} & 0 \\
0 & \bar{\sigma}_{22}^{I I}
\end{array}\right]
$$

$$
\bar{\sigma}_{11}^{I I}=\left(\left(1-c^{I I}\right) / \sigma_{0}^{(3)}+c^{I I} / \bar{\sigma}_{11}^{I}\right)^{-1}, \quad \bar{\sigma}_{22}^{I I}=c^{I I} \bar{\sigma}_{22}^{I}+\left(1-c^{I I}\right) \sigma_{0}^{(3)},
$$

where $\bar{\sigma}_{11}^{I}$ and $\bar{\sigma}_{22}^{I}$ are the diagonal components of the matrix in (63). If component $\sigma_{0}^{(1)}$ occupies overall volume fraction $c^{(1)}$ in this laminate, then $c^{I I} c^{I}=c^{(1)}$. This composite can be made isotropic in the $\left(x_{1}, x_{2}\right)$-plane by selecting the appropriate volume fraction $c^{I}$ (thus $c^{I I}$ ) through the condition $\bar{\sigma}_{11}^{I I}=\bar{\sigma}_{22}^{I I}$; hence the $\bar{\sigma}^{I I}$ may be expressed by

$$
\left[\overline{\boldsymbol{\sigma}}^{I I}\right]=\bar{\sigma}_{0}^{I I} \mathbf{I},
$$

where $\bar{\sigma}_{0}^{I I}$ is the resultant isotropic conductivity $\left(\bar{\sigma}_{0}^{I I}=\bar{\sigma}_{11}^{I I}=\bar{\sigma}_{22}^{I I}\right)$ for the rank-II laminate. The isotropic condition is simply

$$
\left(\frac{1-c^{I I}}{\sigma_{0}^{(3)}}+\frac{c^{I I}}{\bar{\sigma}_{11}^{I}}\right)^{-1}=c^{I I} \bar{\sigma}_{22}^{I}+\left(1-c^{I I}\right) \sigma_{0}^{(3)} \text {. }
$$

This equation can be solved to get $c^{I I}$, which together with $c^{(1)}$ gives $c^{I}$. It has been shown by Lurie and Cherkaev [12], Tartar [23] that if this rank-II laminate is made of two isotropic materials (i.e., $\sigma_{0}^{(2)}=\sigma_{0}^{(3)}$ ), it attains the corresponding two-dimensional $\mathrm{H}-\mathrm{S}$ bounds. Here we define the "inclusion" of a laminate to be the area occupied by phase $1\left(\sigma_{0}^{(1)}\right)$; all other regions are called matrix. If $\sigma_{0}^{(1)}>\sigma_{0}^{(2)}$ $\left(=\sigma_{0}^{(3)}\right)$, then the H-S lower bound is attained. If $\sigma_{0}^{(1)}<\sigma_{0}^{(2)}$, then the H-S upper bound is attained.

As a second example, we slice the rank-I laminate in the direction $\mathbf{n}=[-\cos (\pi / 6)$, $\sin (\pi / 6), 0]$, and laminate it with component 3 in this direction $\mathbf{n}$. The resultant rank-II laminate is shown in Fig. $1(\mathrm{c})$, and its effective electric conductivity $\overline{\boldsymbol{\sigma}}^{I I}$, derived from Eq. (17), has the matrix form

$$
\left[\overline{\boldsymbol{\sigma}}^{I I}\right]=\left[\begin{array}{ll}
\bar{\sigma}_{11}^{I I} & \bar{\sigma}_{12}^{I I} \\
\bar{\sigma}_{21}^{I I} & \bar{\sigma}_{22}^{I I}
\end{array}\right],
$$

where $\bar{\sigma}_{12}^{I I}=\bar{\sigma}_{21}^{I I}$, and

$$
\begin{aligned}
& \bar{\sigma}_{11}^{I I}=\left(1-c^{I}\right) \sigma_{0}^{(3)}+c^{I I} \bar{\sigma}_{11}^{I}-\frac{3}{4} \frac{c^{I I}\left(1-c^{I I}\right)}{D_{1}}\left(\bar{\sigma}_{11}^{I}-\sigma_{0}^{(3)}\right)^{2}, \\
& \bar{\sigma}_{12}^{I I}=\frac{\sqrt{3}}{4} \frac{c^{I I}\left(1-c^{I I}\right)}{D_{1}}\left(\bar{\sigma}_{11}^{I}-\sigma_{0}^{(3)}\right)\left(\bar{\sigma}_{22}^{I}-\sigma_{0}^{(3)}\right), \\
& \bar{\sigma}_{22}^{I I}=\left(1-c^{I I}\right) \sigma_{0}^{(3)}+c^{I I} \bar{\sigma}_{22}^{I}-\frac{1}{4} \frac{c^{I I}\left(1-c^{I I}\right)}{D_{1}}\left(\bar{\sigma}_{22}^{I}-\sigma_{0}^{(3)}\right)^{2},
\end{aligned}
$$

with

$$
D_{1}=c^{I I} \sigma_{0}^{(3)}+\left(1-c^{I I}\right)\left(\frac{3}{4} \bar{\sigma}_{11}^{I}+\frac{1}{4} \bar{\sigma}_{22}^{I}\right) \text {. }
$$


Using the same procedure, a rank-III laminate can be constructed by laminating the above rank-II composite (with relative volume fraction $c^{I I I}$ ) with another isotropic material (with electrical conductivity $\sigma_{0}^{(4)}$ and relative volume fraction $1-c^{I I I}$ ) in the direction $\mathbf{n}=\left[\cos \frac{\pi}{6}, \sin \frac{\pi}{6}, 0\right]$. By using Eq. (17), the effective conductivity $\overline{\boldsymbol{\sigma}}^{I I I}$ is given by

$$
\left[\overline{\boldsymbol{\sigma}}^{I I I}\right]=\left[\begin{array}{ll}
\bar{\sigma}_{11}^{I I I} & \bar{\sigma}_{12}^{I I I} \\
\bar{\sigma}_{21}^{I I I} & \bar{\sigma}_{22}^{I I I}
\end{array}\right],
$$

where $\bar{\sigma}_{12}^{I I I}=\bar{\sigma}_{21}^{I I I}$, and

$$
\begin{gathered}
\bar{\sigma}_{11}^{I I I}=\left(1-c^{I I I}\right) \sigma_{0}^{(4)}+c^{I I I} \bar{\sigma}_{11}^{I I}-\frac{c^{I I I}\left(1-c^{I I I}\right)}{D_{2}}\left[\frac{1}{2} \bar{\sigma}_{12}^{I I}+\frac{\sqrt{3}}{2}\left(\bar{\sigma}_{11}^{I I}-\sigma_{0}^{(4)}\right)\right]^{2}, \\
\bar{\sigma}_{12}^{I I I}=c^{I I I} \bar{\sigma}_{12}^{I I}-\frac{c^{I I I}\left(1-c^{I I I}\right)}{D_{2}} \\
\times\left[\frac{1}{2} \bar{\sigma}_{12}^{I I}+\frac{\sqrt{3}}{2}\left(\bar{\sigma}_{11}^{I I}-\sigma_{0}^{(4)}\right)\right]\left[\frac{\sqrt{3}}{2} \bar{\sigma}_{12}^{I I}+\frac{1}{2}\left(\bar{\sigma}_{22}^{I I}-\sigma_{0}^{(4)}\right)\right], \\
\bar{\sigma}_{22}^{I I I}=\left(1-c^{I I I}\right) \sigma_{0}^{(4)}+c^{I I I} \bar{\sigma}_{22}^{I I}-\frac{c^{I I I}\left(1-c^{I I I}\right)}{D_{2}}\left[\frac{\sqrt{3}}{2} \bar{\sigma}_{12}^{I I}+\frac{1}{2}\left(\bar{\sigma}_{22}^{I I}-\sigma_{0}^{(4)}\right)\right]^{2},
\end{gathered}
$$

with

$$
D_{2}=c^{I I I} \sigma_{0}^{(4)}+\frac{\left(1-c^{I I I}\right)}{4}\left(3 \bar{\sigma}_{11}^{I I}+\bar{\sigma}_{22}^{I I}+2 \sqrt{3} \bar{\sigma}_{12}^{I I}\right) .
$$

Here $c^{I I I} c^{I I} c^{I}=c^{(1)}$, where $c^{(1)}$ is the overall volume fraction of $\sigma_{0}^{(1)}$. This is an anisotropic composite. To make it isotropic we need the diagonal terms to be equal (represented by variable $\bar{\sigma}_{0}^{I I I}$ ) and the off-diagonal terms to vanish. Thus, we have two constraint equations for isotropy:

$$
\bar{\sigma}_{11}^{I I I}=\bar{\sigma}_{22}^{I I I}=\bar{\sigma}_{0}^{I I I} \text { and } \bar{\sigma}_{12}^{I I I}=0 .
$$

The solution of the above equations gives the relative volume fractions $c^{I I I}, c^{I I}$, and $c^{I}$. The isotropic conductivity is then given by

$$
\left[\overline{\boldsymbol{\sigma}}^{I I I}\right]=\bar{\sigma}_{0}^{I I I} \mathbf{I} \text {. }
$$

By choosing $\sigma_{0}^{(2)}=\sigma_{0}^{(3)}=\sigma_{0}^{(4)}$, the above rank-III isotropic laminate has the same effective property with that of the rank-II isotropic laminate expressed in Eq. (67), i.e., $\bar{\sigma}_{0}^{I I}=\bar{\sigma}_{0}^{I I I}$. Both of them again attain the linear H-S bounds in the two-dimensional cases.

(b) Three-dimensional laminates. In the previous subsection, the laminating process is confined to the $\left(x_{1}, x_{2}\right)$-plane, resulting in long fiber microstructures. The properties in the $x_{3}$ direction are not considered. In fact, the properties in the $x_{3}$ direction for these laminates are merely the volume averages of the properties in the $x_{3}$ direction of the components. The three-dimensional laminates discussed in this subsection are constructed based on the two-dimensional laminates by an additional 
laminating in the $x_{3}$ direction. An orthogonal three-dimensional laminate is composed by slicing the rank-II laminate represented by Eq. (65) in the $x_{3}$ direction and then layering it with an isotropic material (with electrical conductivity $\sigma_{0}^{(4)}$ ). The rank-II component occupies the relative volume fraction $c^{I I I}$ and the isotropic component occupies the relative volume fractions $1-c^{I I I}$. The effective electrical conductivity $\overline{\boldsymbol{\sigma}}^{I I I}$ can be obtained from (17) in a diagonal matrix form

$$
\left[\overline{\boldsymbol{\sigma}}^{I I I}\right]=\left[\begin{array}{lll}
\bar{\sigma}_{11}^{I I I} & & \\
& \bar{\sigma}_{22}^{I I I} & \\
& & \bar{\sigma}_{33}^{I I I}
\end{array}\right],
$$

where

$$
\begin{aligned}
& \bar{\sigma}_{11}^{I I I}=\left(1-c^{I I I}\right) \sigma_{0}^{(4)}+c^{I I I} \frac{\left(c^{I} \sigma_{0}^{(1)}+\left(1-c^{I}\right) \sigma_{0}^{(2)}\right) \sigma_{0}^{(3)}}{\left(1-c^{I I}\right)\left(c^{I} \sigma_{0}^{(1)}+\left(1-c^{I}\right) \sigma_{0}^{(2)}\right)+c^{I I} \sigma_{0}^{(3)}}, \\
& \bar{\sigma}_{22}^{I I I}=\left(1-c^{I I I}\right) \sigma_{0}^{(4)}+c^{I I I}\left(1-c^{I I}\right) \sigma_{0}^{(3)}+c^{I I I} c^{I I} \frac{\sigma_{0}^{(1)} \sigma_{0}^{(2)}}{c^{I} \sigma_{0}^{(2)}+\left(1-c^{I}\right) \sigma_{0}^{(1)}}, \\
& \bar{\sigma}_{33}^{I I I}=\frac{\sigma_{0}^{(4)}\left[\left(1-c^{I I}\right) \sigma_{0}^{(3)}+c^{I I}\left(c^{I} \sigma_{0}^{(1)}+\left(1-c^{I}\right) \sigma_{0}^{(2)}\right)\right]}{\left(1-c^{I I I}\right)\left[\left(1-c^{I I}\right) \sigma_{0}^{(3)}+c^{I I}\left(c^{I} \sigma_{0}^{(1)}+c_{2}^{I} \sigma_{0}^{(2)}\right)\right]+c^{I I I} \sigma_{0}^{(4)}} .
\end{aligned}
$$

If $\sigma_{0}^{(1)}$ occupies volume fraction $c^{(1)}, c^{I I I} c^{I I} c^{I}=c^{(1)}$. This laminate can be made isotropic by choosing appropriate relative volume fractions $c^{I}, c^{I I}$, and $c^{I I I}$. This can be accomplished by equating the diagonal terms of (79), i.e.,

$$
\bar{\sigma}_{11}^{I I I}=\bar{\sigma}_{22}^{I I I}=\bar{\sigma}_{33}^{I I I}=\bar{\sigma}_{0}^{I I I} .
$$

Here $\bar{\sigma}_{0}^{I I I}$ is the resultant effective conductivity of the three-dimensional laminate. If Eq. (81) is satisfied, the resulting isotropic effective conductivity can be written

$$
\left[\overline{\boldsymbol{\sigma}}^{I I I}\right]=\bar{\sigma}_{0}^{I I I} \mathbf{I} \text {. }
$$

In the above three-dimensional isotropic laminates, a two-phase composite is constructed if the matrix is composed of the same material (i.e., $\sigma_{0}^{(2)}=\sigma_{0}^{(3)}=\sigma_{0}^{(4)}$ ). Its conductivity coincides with the three-dimensional linear H-S bounds. Again, the upper bound is reached when the inclusions (noncontinuous phase) are less conductive then the matrix (continuous phase) material; the lower bound is attained when the inclusions are more conductive than the matrix.

Appendix B. Nonlinear H-S bounds. By restricting $\sigma_{0}(\mathbf{x})$ to be piecewise constant in each phase $\left(\sigma_{0}(\mathbf{x})=\sigma_{0}^{(r)}\right.$ in phase $\left.r\right)$ of a general $N$-phase isotropic composite, Eq. (15) becomes an inequality [1], since the maximum is over a smaller class of functions (functions that are piecewise constant) than the class of nonnegative functions to which $\sigma_{0}(\mathbf{x})$ actually belongs. Thus, Eq. (15) leads to

$$
\widetilde{W}(\overline{\mathbf{E}}) \geq \max _{\sigma_{0}^{(r)}>0}\left\{\widetilde{W}_{0}(\overline{\mathbf{E}})-\sum_{r=1}^{N} c^{(r)} v^{(r)}\left(\sigma_{0}^{(r)}\right)\right\},
$$


where $\widetilde{W}_{0}$ now represents a linear, isotropic comparison composite with linear conductive constants $\sigma_{0}^{(r)}$ in volume fractions $c^{(r)}$, and $v^{(r)}$ is given by (21). The effective conductivity of the linear comparison material, $\bar{\sigma}_{0}$, is bounded by the linear H-S lower and upper bounds [3], $\bar{\sigma}_{-}$and $\bar{\sigma}_{+}$respectively; thus,

$$
\bar{\sigma}_{-} \leq \bar{\sigma}_{0} \leq \bar{\sigma}_{+} .
$$

These H-S bounds are given by (see Lurie and Cherkaev [12] or Kohn and Milton [21])

$$
\begin{aligned}
& \bar{\sigma}_{-}=(1-d) \sigma_{-}+\left(\sum_{r=1}^{N} \frac{c^{(r)}}{\sigma_{0}^{(r)}+(d-1) \sigma_{-}}\right)^{-1}, \\
& \bar{\sigma}_{+}=(1-d) \sigma_{+}+\left(\sum_{r=1}^{N} \frac{c^{(r)}}{\sigma_{0}^{(r)}+(d-1) \sigma_{+}}\right)^{-1},
\end{aligned}
$$

where $d(=2,3)$ is the number of space dimensions, and $\sigma_{-}=\min _{r}\left\{\sigma_{0}^{(r)}\right\}$ and $\sigma_{+}=\max _{r}\left\{\sigma_{0}^{(r)}\right\}$ correspond to the conductivity of the least and most conductive phases respectively.

If an extremal comparison material with minimum linear isotropic conductivity $\bar{\sigma}_{-}$is introduced into Eq. (83), the linear comparison material is characterized by $\widetilde{W}_{0}=(1 / 2) \bar{\sigma}_{-} \bar{E}^{2}$. Since this is the smallest possible comparison energy function (see Eq. (84)), a strict lower bound (denoted $W_{\mathrm{HS}_{-}}$) for nonlinear composites will be generated by substituting this $\widetilde{W}_{0}$ into (83). On the other hand, if an extremal material with maximum linear conductivity $\bar{\sigma}_{+}$is introduced, the largest comparison energy, $\widetilde{W}_{0}=(1 / 2) \bar{\sigma}_{+} \bar{E}^{2}$, is obtained. However, because of the inequality in (83) only an estimate for the corresponding nonlinear upper bound is obtained. We write this estimate to the nonlinear upper bound $W_{\mathrm{HS}_{+}}$.

It should be noted that the effective properties of nonlinear isotropic composites depend on the applied electric field intensity $\bar{E}$. The properties of the optimal comparison materials, $\sigma_{0}^{(r)}$, must also change with $\bar{E}$. Thus the maximum and minimum conductivities $\sigma_{-}$and $\sigma_{+}$also change with $\bar{E}$. In the case of a two-phase composite, nonlinear H-S bounds are derived from linear H-S bounds by substituting either (85) or (86) into (83), to give the effective potential

$$
W_{\mathrm{HS}}(\overline{\mathbf{E}})=\max _{\sigma_{0}^{(1)}, \sigma_{0}^{(2)}}\left[\frac{1}{2}\left(\bar{\sigma}_{0}\right)_{i} \bar{E}^{2}-\sum_{r=1}^{2} c^{(r)} v^{(r)}\left(\sigma_{0}^{(r)}\right)\right] .
$$

When $\left(\bar{\sigma}_{0}\right)_{i}$ is defined by

$$
\left(\bar{\sigma}_{0}\right)_{i}=(1-d) \sigma^{(1)}+\left(\sum_{r=1}^{2} \frac{c^{(r)}}{\sigma_{0}^{(r)}+(d-1) \sigma_{0}^{(1)}}\right)^{-1} \text {, then } W_{\mathrm{HS} 1}=W_{\mathrm{HS}},
$$

and when

$$
\left(\bar{\sigma}_{0}\right)_{i}=(1-d) \sigma^{(2)}+\left(\sum_{r=1}^{2} \frac{c^{(r)}}{\sigma_{0}^{(r)}+(d-1) \sigma_{0}^{(2)}}\right)^{-1}, \text { then } W_{\mathrm{HS} 2}=W_{\mathrm{HS}} .
$$


The H-S lower bound and the H-S estimate for the upper bound are then given by the maximum and minimum of the two nonlinear bounds $W_{\mathrm{HS} 1}$ and $W_{\mathrm{HS} 2}$, namely

$$
W_{\mathrm{HS}-}=\min \left(W_{\mathrm{HS} 1}, W_{\mathrm{HS} 2}\right) \text { and } W_{\mathrm{HS}+}=\max \left(W_{\mathrm{HS}_{1}}, W_{\mathrm{HS}_{2}}\right) \text {. }
$$

For more details, the reader is referred to $[1,14]$.

\section{REFERENCES}

[1] P. Ponte Castañeda, The effective mechanical properties of nonlinear isotropic composites, J. Mech. Phys. Solids 39, 45 (1991)

[2] J. C. Maxwell, Electricity and magnetism, 1st ed., Clarendon, Oxford, 1873

[3] Z. Hashin and S. Shtrikman, $A$ variational approach to the theory of the effective magnetic permeability of multiphase materials, J. Appl. Phys. 33, 3125 (1962)

[4] E. H. Kerner, The elastic and thermoelastic properties of composite media, Proc. Phys. B 69, 802 (1956)

[5] J. R. Willis, Bounds and self-consistent estimates for the overall properties of anisotropic composites, J. Mech. Phys. Solids 25, 185 (1977)

[6] R. Landauer, Electrical conductivity in inhomogeneous media, in Electrical Transport and Optical Properties of Inhomogeneous Media, ed. J. C. Garland and D. B. Tanner, American Institute of Physics, New York, 1978

[7] Z. Hashin, Assessment of the self-consistent scheme approximation: Conductivity of particulate composites, J. Compos. Mater. 2, 284 (1968)

[8] D. A. G. Bruggeman, Ph.D. Thesis, Utrecht, 1930

[9] D. A. G. Bruggeman, Berechnung verschiedener physikalischer Konstanten von heterogenen Substanzen. I. Dielektrizitätskonstanten und Leitfähigkeiten der Mischkörper aus isotropen Substanzen, Ann. Phys. 5, 636 (1935)

[10] G. W. Milton, Modeling the properties of composites by laminates, in Homogenization and Effective Moduli of Materials and Media, ed. J. L. Ericksen et al, Springer-Verlag, New York, 1986

[11] G. W. Milton, Concerning bounds on the transport and mechanical properties of multicomponent composite materials, Appl. Phys. A 26, 125 (1981)

[12] K. A. Lurie and A. V. Cherkaev, Exact estimates of conductivity of composites formed by two isotropically conducting media taken in prescribed proportion, Proc. Roy. Soc. Edinburgh A 99, 71-87 (1984)

[13] K. A. Lurie and A. V. Cherkaev, Exact estimates of the conductivity of a binary mixture of isotropic materials, Proc. Roy. Soc. Edinburgh A 104, 21-38 (1986)

[14] P. Ponte Castañeda, Bounds and estimates for the properties of nonlinear heterogeneous systems, Proc. Roy. Soc. Lond. A 340, 531 (1992)

[15] X. C. Zeng, P. M. Hui, D. J. Bergman, and D. Stroud, Mean field theory for weakly nonlinear composites, Phys. A 157, 192 (1989)

[16] J. R. Willis, Variational estimates for the overall response of an inhomogeneous nonlinear dielectric, in Homogenization and Effective Moduli of Materials and Media, ed. J. L. Ericksen et al, Springer-Verlag, New York, 1986

[17] D. R. S. Talbot and J. R. Willis, Bounds and self-consistent estimates for the overall properties of nonlinear composites, IMA J. Appl. Math. 39, 215 (1987)

[18] D. Stroud and P. M. Hui, Nonlinear susceptibilities of granular media, Phys. Rev. B 37, 8719 (1988)

[19] R. Hill, Elastic properties of reinforced solids: Some theoretical principles, J. Mech. Phys. Solids 11, 357 (1963)

[20] L. D. Landau, E. M. Lifshitz, and L. P. Pitaevskii, Electrodynamics of Continuous Media, 2nd edition, Pergamon, Oxford, 1984

[21] R. V. Kohn and G. W. Milton, Variational bounds on the effective moduli of anisotropic composites, J. Mech. Phys. Solids 36, 597 (1988)

[22] R. Blumenfeld and D. J. Bergman, Nonlinear dielectric: Electrostatics of random media and propagation of electromagnetic waves in a homogeneous slab, Phys. A 157, 428 (1989)

[23] L. Tartar, Estimations fines de coefficients homogénéisés, Research Notes in Mathematics 125, 168 (1985) 